Nora Ketschik, André Blessing, Sandra Murr, Maximilian Overbeck und Axel Pichler

\title{
Interdisziplinäre Annotation von Entitätenreferenzen
}

\author{
Von fachspezifischen Fragestellungen zur einheitlichen \\ methodischen Umsetzung
}

Zusammenfassung: Der Beitrag präsentiert die Ergebnisse der interdisziplinären Auseinandersetzung mit Entitäten. Wir erarbeiten disziplinübergreifende Richtlinien für eine reliable und semantisch valide Annotation von Entitätenreferenzen sowie einen gemeinsamen methodischen Workflow für eine semi-automatische Klassifikation großer Textmengen. Aus vier sozial- und geisteswissenschaftlichen Perspektiven heraus diskutieren wir anschließend Herausforderungen und Lösungsmöglichkeiten, die bei der Anwendung unseres Ansatzes auf die jeweiligen fachspezifischen Textkorpora auftreten. Wir zeigen, dass die interdisziplinäre Kooperation zur methodischen Stringenz verpflichtet und zu einer reflexiven Entwicklung der Analysekonzepte und Operationalisierungsprozesse beiträgt.

Abstract: This contribution presents the results of our interdisciplinary engagement with entities. We developed interdisciplinary guidelines for a reliable and semantically valid annotation of entity references, and a methodological workflow for their semi-automatic identification within large amounts of textual data. From the perspective of four different disciplines within the Humanities and Social Sciences, we discuss challenges related to the application of a generic workflow to heterogeneous text corpora, and present possible solutions. We conclude that the interdisciplinary collaboration enhances the overall methodological stringency and fosters conceptual reflections within each participating discipline.

Nora Ketschik, Institut für Literaturwissenschaft, Universität Stuttgart

Maximilian Overbeck, Department of Communication and Journalism, Hebrew University of Jerusalem

Sandra Murr, Axel Pichler, Stuttgart Research Center for Text Studies, Universität Stuttgart André Blessing, Institut für Maschinelle Sprachverarbeitung, Universität Stuttgart

Ә Open Access. (c) 2020 Nora Ketschik, André Blessing, Sandra Murr, Maximilian Overbeck und Axel Pichler; publiziert von De Gruyter (c) BY-NC-ND Dieses Werk ist lizenziert unter der Creative Commons AttributionNonCommercial-NoDerivatives 4.0 International Lizenz.

https://doi.org/10.1515/9783110693973-010 


\section{Einleitung}

In diesem Beitrag präsentieren wir die Resultate der interdisziplinären methodischen Auseinandersetzung mit Entitäten und deren Referenzen, die im Rahmen des DH-Projekts CRETA erzielt wurden. ${ }^{1}$ Ursprünglich stammt der Begriff ,Entität‘ aus der Philosophie und zwar aus der allgemeinen Metaphysik bzw. Ontologie, die die elementaren Grundstrukturen der Wirklichkeit untersucht. Hierin bezeichnet er ein einzelnes unteilbares Seiendes. Entitäten spielen insbesondere in den Kategorienlehren eine zentrale Rolle, im Rahmen derer die Philosophie seit der Antike den Gesamtbereich des Seienden zu klassifizieren versucht. ${ }^{2}$ Derartige Klassifikationspraktiken sind auch in anderen Disziplinen relevant. So untersucht die Politikwissenschaft beispielsweise, wie in den verschiedenen Arenen des politischen und gesellschaftlichen Lebens auf Entitäten wie politische Akteure, Parteien oder Organisationen referiert wird. Für die germanistische Mediävistik und die Neuere Deutsche Literatur steht die Erfassung von Referenzen auf Figuren und deren Relationen zueinander in den fachspezifischen Texten im Fokus. Im Rahmen von CRETA wurde ein Umgang mit Entitäten und deren Referenzen angestrebt, der für Fragestellungen aus den unterschiedlichsten Fächern von Interesse und Nutzen sein sollte. Im Unterschied zur named entity recognition (NER), die das Ziel verfolgt, Eigennamen im Text zu erkennen (Jurafsky und Martin 2008, S. 793-746; Carstensen et al. 2010, S. 596-599; Clark et al. 2013, S. 518-522) ${ }^{3}$ sind für uns neben Eigennamen auch Gattungsnamen von Interesse. Zudem erweitern wir das Repertoire der gängigen Entitätenklassen PER (Personen, Figuren), LOC (Orte) und ORG (Organisationen) um die Klassen WRK (Werke) und CNC (Abstrakte Konzepte). Im Kontext der hier vorgestellten Projekte bezeichnet ,Entität‘ folglich isolierte reale, fiktive oder mögliche Objekte, auf die von der Textoberfläche mittels eines einzelnen Wortes oder einer abgegrenzten Wortfolge referiert werden kann.

Während sich einzelne Disziplinen aus unterschiedlichen inhaltlichen Gesichtspunkten für Entitäten interessieren, lassen sich auf der Oberfläche eines Textes nur Entitätenreferenzen erfassen, also diejenigen sprachlichen Ausdrücke, die auf eine bestimmte Entität in der realen, fiktiven oder einer möglichen Welt

1 Siehe dazu auch Kuhns Einleitung des Bandes ab Seite 9.

2 Seit dem 19. Jahrhundert herrscht in der Philosophie Skepsis darüber, ob philosophische Kategorienlehren ihre Minimalanforderungen - d. s. die Vollständigkeit und Exaktheit der Kategorien - erfüllen bzw. welche Kriterien zur Überprüfung dieses Sachverhaltes anzuwenden sind. Dies bedeutet jedoch nicht, dass die Philosophie gegenwärtig auf die Arbeit an Kategoriensystemen verzichtet.

3 Die NER wurde v. a. durch die Message Understanding Conferences (MUC) 6 und 7 (1994-1998) populär. 
referieren. Die Annotation von Entitätenreferenzen erfolgt im Rahmen von CRETA vor dem Hintergrund verschiedener Forschungsfragen und in Textkorpora unterschiedlicher Disziplinen: in einem sozialwissenschaftlichen Korpus bestehend aus Plenardebatten des deutschen Bundestags, in einer Sammlung von WertherAdaptionen und ihrer Vorlage, Goethes Die Leiden des jungen Werthers, in einem altgermanistischen Korpus mittelhochdeutscher Artusromane, sowie in Schriften zur philosophischen Ästhetik des 20. Jahrhunderts.

Die Auseinandersetzung mit Entitäten und deren Referenzen stellte ein übergreifendes Forschungsinteresse aller an CRETA beteiligten Fächer dar und wurde bewusst an den Anfang des Förderzeitraums gesetzt, um eine gemeinsame Grundlage für darauf aufbauende Analysen zu schaffen. Zudem sollte sie dazu beitragen, disziplinäre Fragestellungen weiterzuentwickeln und zu präzisieren. ${ }^{4}$

Die Struktur unseres Beitrags orientiert sich an der Chronologie unseres interdisziplinären Workflows, so wie er bei der Analyse der Entitäten und ihrer textuellen Referenzen entstanden ist. Im ersten Abschnitt gehen wir ausführlich auf die gemeinsame Entwicklung der Annotationsrichtlinien ein. Mit den Richtlinien wird das Ziel verfolgt, die im Rahmen von CRETA untersuchten Entitätenklassen auf Basis valider Textindikatoren zu erfassen. Wir veranschaulichen den disziplinübergreifenden Workflow, der qualitative und quantitative Analyseschritte systematisch miteinander kombiniert und die Identifikation von Entitätenreferenzen zunächst auf kleinen und anschließend großen Textkorpora anleitet. Im zweiten Abschnitt gehen wir genauer auf die vier geistes- und sozialwissenschaftlichen Zugänge zu Entitäten ein und widmen uns kritisch den Problemen und Herausforderungen, die bei der Erfassung und Analyse von Entitätenreferenzen in unterschiedlichen Textkorpora entstanden sind. Im dritten Abschnitt fassen wir in einer Schlussbetrachtung unsere Forschungsergebnisse zusammen.

\section{Interdisziplinärer Austausch und gemeinsame methodische Umsetzung}

\subsection{Annotationsrichtlinien}

Am Beginn des interdisziplinären Annotationsprozesses stand die gemeinsame Entwicklung fachübergreifender Annotationsrichtlinien. Sie wurden im dialogi-

4 Siehe zu interdisziplinärer Kommunikation auch den Beitrag „Reaching out: Interdisziplinäre Kommunikation und Dissemination“ ab Seite 467 (Reiter, Kremer et al. 2020). 
schen Austausch und auf der Basis fachspezifischen Wissens über die ausgewählten Textkorpora und deren jeweilige Spezifika entwickelt. ${ }^{5}$ Grundlegend für uns war dabei zunächst, einen gemeinsamen Zugriff auf Entitäten und ihre Referenzen zu erarbeiten. Wir gingen dabei von der NER aus und orientierten uns an den gängigen Annotationspraktiken aus der Computer- und Korpuslinguistik. Im Zuge der interdisziplinären Erarbeitung von Annotationsrichtlinien erwies sich für die in CRETA versammelten Disziplinen eine Entitäten-Konzeption als sinnvoll, die sich zwischen der Eigennamenerkennung (NER, Jurafsky und Martin 2008, S. 739-746; Carstensen et al. 2010, S. 596-599; Clark et al. 2013, S. 518-522) und der Koreferenzresolution (Jurafsky und Martin 2008, S. 694, 707-724; Clark et al.2013, S. 522f.) bewegt: Die CRETA-Annotationsrichtlinien gehen über die reine Eigennamenerkennung hinaus, da auch Entitätenreferenzen via Gattungsnamen berücksichtigt werden, sie bleiben aber hinter einer vollständigen Koreferenzresolution zurück, da weder pronominale Referenzen erfasst noch alle Entitätenreferenzen zu Koreferenzketten gruppiert werden.

Neben solch grundlegenden Entscheidungen hatten die Richtlinien vor allem den Zweck, präzise Handlungsanweisungen für das Annotieren von Entitäten zu formulieren: So geben die CRETA-Annotationsrichtlinien z. B. vor, immer syntaktische Einheiten, also maximale Nominalphrasen zu annotieren, so dass auch Artikel, Adjektive, Appositionen und Relativsätze Teil des Referenzausdrucks sein können (z. B. „meine liebste Lotte, mein Engel“). Dies ist zum einen mit der gängigen Praxis in der Computerlinguistik konform (Reznicek 2013a,b) und hat zum anderen den Grund, dass semantische, die Entität näher bestimmende Informationen für weiterführende Analysen miterfasst werden. Durch die Annotation der maximalen Nominalphrase kann es zu ,Verschachtelungen` von Entitätenreferenzen kommen, wobei immer alle Referenzausdrücke - alle eingebetteten wie der maximale - zu annotieren sind (z. B. ,[Angela Merkel, die Kanzlerin [der Bundesrepublik Deutschland]]"). Darüber hinaus wird eine Entitätenreferenz immer in ihrem jeweiligen semantischen Kontext interpretiert und annotiert, um eventuelle Ambiguitäten aufzulösen - z. B. kann sich „Washington“ je nach Kontext auf einen Ort (LOC), eine Organisation (ORG, hier die US-Regierung), oder auf die Person (PER, ,George Washington') beziehen.

Generell wird in CRETA eine Vollannotation angestrebt, was bedeutet, dass alle Entitätenreferenzen in einem Text unabhängig von ihrem Status oder ihrer Relevanz annotiert werden. So kann für den späteren Einsatz maschineller Lernverfahren eine vollständige Datengrundlage geschaffen werden. Die Vollannotation

5 Unsere Annotationsrichtlinien orientieren sich grob an Reznicek (2013b) und Reznicek (2013a). Die vollständigen Annotationsrichtlinien sind im Anhang beigefügt. 
macht es notwendig, gegebenenfalls in einem zweiten Schritt über die Relevanz einer Entitätenreferenz für die jeweilige Forschungsfrage zu urteilen oder weitere Merkmale zu ihrer Differenzierung (z. B. die Unterscheidung von historischer Person und fiktiver Figur) hinzuzufügen.

Die Annotationsrichtlinien werden durch Mitarbeitende in einer ersten Version aufgesetzt und an Textbeispielen der verschiedenen Disziplinen geprüft. In regelmäßigen Projekttreffen werden die Anwendungsfälle besprochen und die Richtlinien erweitert, präzisiert und korrigiert. Durch die direkte Anwendung werden sehr praxisnahe Richtlinien erstellt, die einerseits durch Beispiele konkretisiert, andererseits durch die heterogenen Beispieltexte allgemein gehalten werden und so für verschiedene Disziplinen nutzbar sind.

Ein wesentlicher Beitrag des interdisziplinären Vorgehens liegt in der Entwicklung eines Workflows für die transparente und intersubjektiv nachvollziehbare manuelle Annotation der Referenzausdrücke. Die zentrale Herausforderung besteht darin, die Replizierbarkeit der Annotationen zu ermöglichen, so dass unterschiedliche Annotierende mit derselben Annotationsrichtlinie zu einheitlichen Annotationsergebnissen gelangen. Darüber hinaus sollen die annotierten Referenzausdrücke die Entitätentypen semantisch valide abbilden.

\subsection{Technische Umsetzung und Workflow}

\subsubsection{Entitätenannotation in CRETAnno}

Die Annotation der Entitätenreferenzen wird in einem eigens für das Projekt entwickelten Annotationstool - CRETAnno ${ }^{6}$ - vorgenommen, das den Annotierenden über eine intuitive graphische Benutzeroberfläche die Möglichkeit gibt, mittels Markierung einen Textausschnitt (ein oder mehrere Wörter) als Entitätenreferenz auszuzeichnen (Abbildung 1). Bei der Auszeichnung wird über ein ,Pop-up'Fenster zugleich die Entitätenklasse festgelegt, der die Entität angehört. Bei Unklarheiten kann die Kategorie ,Wiedervorlage‘ angewählt werden, die es erlaubt, die problematischen Fälle zu kommentieren und sie in einem späteren Schritt nochmals separat anzeigen zu lassen.

Für die Inspektion paralleler Annotationen ein und derselben Textstelle durch mehrere Annotierende steht darüber hinaus eine spezielle Ansicht (GoldOverview) zur Verfügung. Sie hebt nicht-übereinstimmende Annotationen hervor

6 http://hdl.handle.net/11022/1007-0000-0007-E1BE-5 


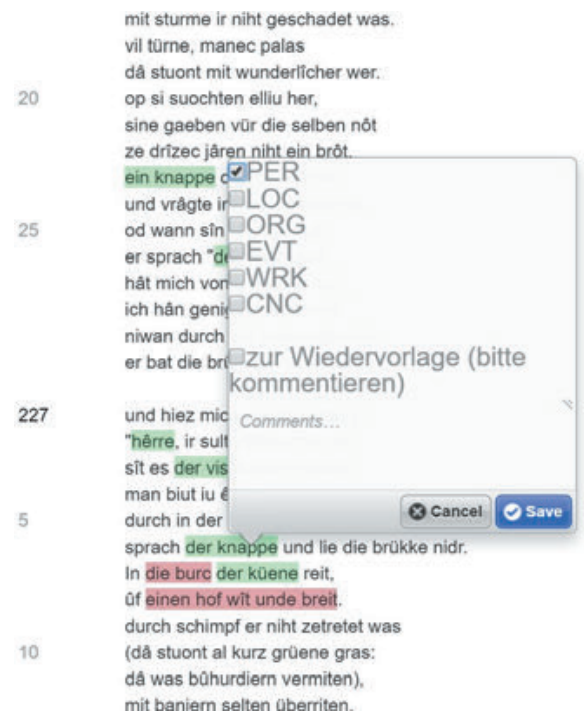

Abb. 1: Benutzeroberfläche zur Entitätenannotation in CRETAnno

und gibt den Annotierenden so die Möglichkeit, diese Fälle zu diskutieren und infolgedessen eine der Annotationen als korrekt festzulegen (Abbildung 2).

\subsubsection{Manueller Annotationsprozess}

Der Arbeitsablauf zur manuellen Annotation ist in Abbildung 3 zusammengefasst. Die Annotationsrichtlinien werden zunächst einem iterativen Prozess aus Anwendung und Überarbeitung unterzogen, indem a) die Richtlinien händisch durch mehrere Annotierende auf ausgewählte Textstellen angewendet (Parallelannotation), b) die Annotationen anschließend verglichen und c) die Richtlinien entsprechend überarbeitet werden. ${ }^{7}$ Bei diesem wiederholenden Vorgehen entstehen verschiedene Versionen der Richtlinien, die aufeinander aufbauen und schließlich in einer finalen Version münden. Für die Überarbeitung der Richtlinien erweist sich der systematische Abgleich nicht-übereinstimmender Annotationen als fruchtbar, da dieser die ,Schwachstellen' der Richtlinien - etwa unzureichende Präzision oder nicht bedachte Fälle - offenlegt. Die vergleichende Parallelannotation wird so lange fortgeführt, bis die Entitätenreferenzen adäquat erfasst und eine möglichst hohe Übereinstimmung zwischen den Annotationen erzielt wird. Der zykli-

7 Vgl. hierzu auch die Anleitung zur Erstellung von Richtlinien von Reiter (2020), ab Seite 193 in diesem Band 


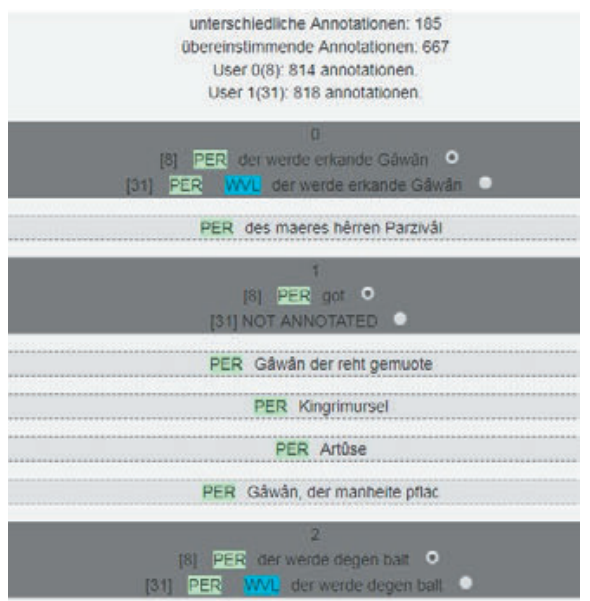

Abb. 2: Gold-Overview in CRETAnno mit Auflistung übereinstimmender und nichtübereinstimmender Annotationen

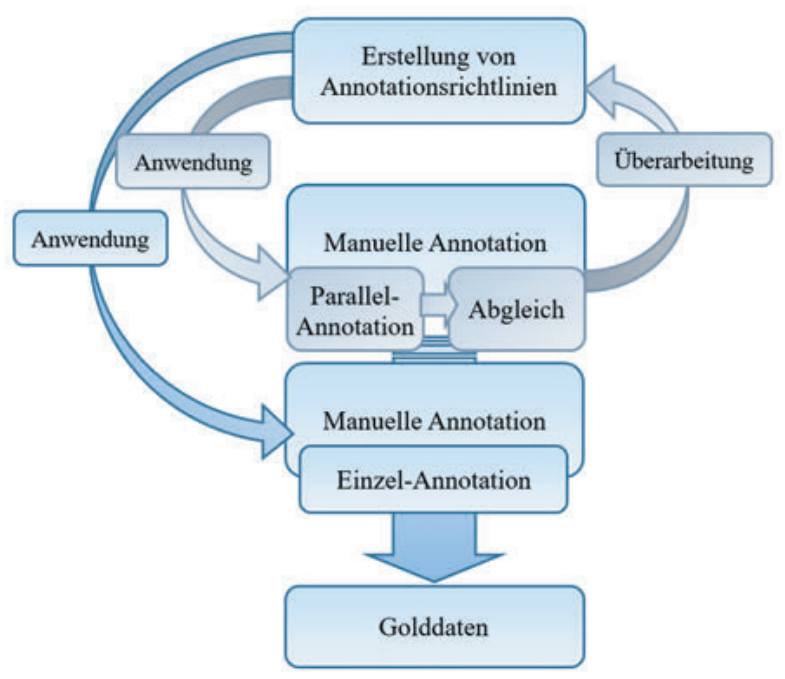

Abb. 3: Workflow zur manuellen Annotation 
sche Prozess verfolgt damit zum einen den Zweck, Unklarheiten in den Richtlinien zu beseitigen; zum anderen schult die wiederholte Anwendung die Annotierenden und befähigt sie, die Richtlinie anschließend autonom auf neue Texte anzuwenden, so dass im Anschluss an die Parallelannotation die Annotation in Einzelarbeit fortgeführt werden kann.

Die intensive manuelle Annotation führt nicht nur zu einer Schärfung der verwendeten Kategorien und Konzepte, sondern sie deckt auch text- bzw. korpusspezifische Besonderheiten auf. Das hat zur Folge, dass die unterschiedlichen Teilgruppen die generischen Annotationsrichtlinien stellenweise genauer an ihre jeweiligen Textsorten anpassen müssen. Für die Annotation des sozialwissenschaftlichen Plenardebatten-Korpus wurden z. B. Zusatzrichtlinien und Beispiele eingefügt, die die Disambiguierung zwischen Orten und Organisationen erleichtern; für die fiktionalen Texte in den literaturwissenschaftlichen Korpora wird die Annotation von Orten weiter geschärft und für die mittelhochdeutschen Artusromane muss zusätzlich über die Nennung von Gott, Heiligen oder menschenähnlichen Handlungsträgern (Riesen, Zwerge, Feen) entschieden werden. Darüber hinaus machen sprachliche Besonderheiten des Mittelhochdeutschen (z. B. Wortverschmelzungen) es notwendig, die Grenzen des zu annotierenden Ausdrucks genauer zu bestimmen. Insgesamt wird der disziplinübergreifende Zugriff auf Entitäten also um disziplinspezifische Zusätze ergänzt, die den Besonderheiten der jeweiligen Korpora Rechnung tragen.

\subsubsection{Semi-automatischer Annotationsprozess}

Durch die händische Annotation der Entitätenreferenzen in den verschiedenen Korpora werden hochqualitative Daten (sog. ,Golddaten') erzeugt, die daraufhin zum Training eines Entitätenreferenzerkenners (ERT) verwendet werden. Während die Annotationsrichtlinien für die Entitätenannotation disziplinübergreifend entwickelt werden können, erweist es sich für den maschinellen Lernprozess als sinnvoll, disziplinspezifische Modelle zu trainieren, so dass das Vorhersagemodell die Besonderheiten der jeweiligen Texte erlernen kann (vgl. Schulz 2018, S. 35-39).

Abbildung 4 beschreibt die Vorgehensweise der semi-automatischen Annotation, die im Anschluss an die händische Annotation angestoßen wird: Basierend auf den manuell annotierten Daten einer bestimmten Disziplin wird ein Entitätenreferenztagger trainiert, der Vorhersagen über Entitätenreferenzen in neuen Texten derselben Disziplin generiert. Daraufhin werden die automatisch erzeugten Annotationen einem Korrekturprozess unterworfen, bei dem die Annotationen als korrekt oder falsch bestimmt und darüber hinaus mögliche fehlende Entitäten- 


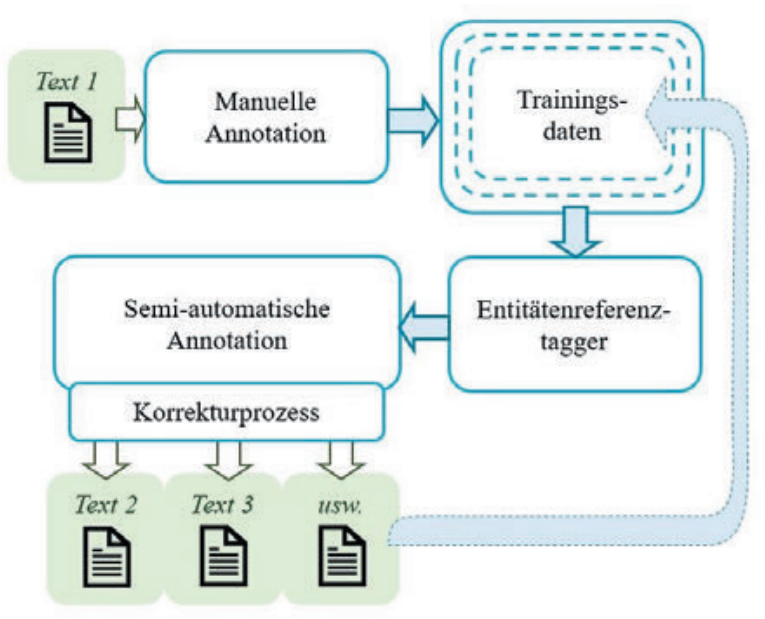

Abb. 4: Workflow zur semi-automatischen Annotation

ze tal gein sinen vuozen nider. der wart schier of gehaben sider do danct or dem markis.

und sprach alsó, daz al sin pris mit der tat waere besiozzen,

und sin triwe mit lobe begozzen,

des sin saelde immer bluete

und $\sin$ unverswigeniu guete

Matribleiz sprach aber me

'unser wer und unser gote hêr

halt niht, wirn muesen unverholen

10 die waren schumptentiure dolen.

daz unsor viuht io wart gesehen.

des mac min herze unsanfte jehen.

min werder got Kahữn wol weiz

sin dienstman Matribleiz

wart zer vluht nie geborn:

ich was ie wol zer wer erkom

giht es daz getoufte her

ich wart ergriffen an der wer

und in Larkant gedrungen,

20

mir No commont

sit !

het foer marcalive]

des richtig akzeptiert (falscher span)

uns faisch falsche Klasse PER

Der marcráve tete im kunt

um einen senlichen vunt, semi-automatische Annotation

- 704 automatische Annotationen

- 326 unbestatigte Annotationen

- $239^{\prime}$ ichifigó Annotationen

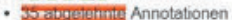

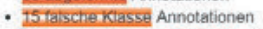

- 89 akzeptierte (falscher spañ) Annotationen

Abb. 5: Benutzeroberfläche zur semi-automatischen Annotation in CRETAnno 
Tab. 1: Anzahl der Tokens und Entitätenreferenzen in den manuell annotierten CRETASubkorpora.

\begin{tabular}{lrr}
\hline Subkorpus & Tokens & Entitäten \\
\hline Literaturwissenschaft (Werther) & 41505 & 331 \\
Philosophie (Adorno) & 13233 & 929 \\
Mediävistik (Parzival) & 30491 & 2001 \\
Sozialwissenschaft (Bundestagsdebatten) & 6371 & 488 \\
\hline
\end{tabular}

\begin{tabular}{lc}
\hline Korpus & Cohen's $\mathbf{~}$ \\
\hline Literaturwissenschaft (Werther) & 0,66 \\
Philosophie (Adorno) & 0,89 \\
Mediävistik (Parzival) & 0,8 \\
Sozialwissenschaft (Bundestagsdebatten) & 0,78 \\
\hline
\end{tabular}

Tab. 2: IAA-Werte (Cohen's $\mathrm{k}$ ) in den parallel annotierten CRETASubkorpora

referenzen händisch nachannotiert werden (Abbildung 5). Auf diese Weise kann die Qualität der Daten gewährleistet werden, so dass diese anschließend zum (Re)Trainieren verwendet werden können. Der sukzessive Aufbau der Trainingsdaten bietet den Vorteil, den Entitätenreferenzerkenner nach und nach auf neue Texte anwenden zu können und hierbei einem Absinken der Performance entgegenzuwirken, die typischerweise aus der Divergenz zwischen Quell- und Zieldaten hervorgeht.

Durch die semi-automatische Vorgehensweise wird einerseits eine erhebliche Zeitersparnis gegenüber der händischen Annotation erzielt, andererseits aber die hohe Qualität der Daten sichergestellt, so dass die anschließenden Untersuchungen auf einer adäquaten Datengrundlage aufbauen können.

\subsection{Korpusstatistik und Evaluation}

Die Modelle des Entitätenreferenzerkenners basieren auf heterogenen Daten, die in Hinblick auf den Umfang der Tokens und Entitäten stark variieren können. Tabelle 1 gibt einen Überblick über die Größe der manuell annotierten Korpora.

Aus den parallel annotierten Textausschnitten lässt sich ein inter-annotator agreement (IAA) errechnen, das die übereinstimmenden Annotationen in ein Verhältnis zu den nicht-übereinstimmenden setzt und damit einen Richtwert für die Klarheit und Intersubjektivität der Guidelines sowie für die Reproduzierbarkeit der Annotationen darstellt (vgl. Carstensen et al. 2010, S. 152). In unserem Fall wird das IAA-Maß Cohen's $\kappa$ (Cohen 1960) verwendet. Generell wird bei einem Wert von $\kappa \leq 0,8$ eine hohe Reliabilität angenommen. Aus den Subkorpora des 
Tab. 3: Evaluation des Entitätenreferenztaggers (ERT) im Vergleich zur NER-baseline

\begin{tabular}{llcccccc}
\hline Klasse & Korpus & \multicolumn{3}{c}{ NER (baseline) } & \multicolumn{3}{c}{ ERT } \\
& & precision & recall & $\mathbf{F}_{1}$ & precision & recall & $\mathbf{F}_{1}$ \\
\hline \multirow{2}{*}{ PER } & Bundestagsdebatten & 31,58 & 0,31 & 0,61 & 40,62 & 0,67 & 1,32 \\
& Adorno & 35,34 & 2,42 & 4,53 & 45,45 & 0,52 & 1,03 \\
& Parzival & 42,86 & 0,46 & 0,91 & 63,24 & 21,56 & 32,16 \\
& Werther & 59,09 & 5,36 & 9,83 & 65 & 12,07 & 20,26 \\
& & & & & & & \\
\multirow{2}{*}{ LOC } & Bundestagsdebatten & 33,82 & 3,48 & 6,31 & 72,22 & 1,97 & 3,89 \\
& Parzival & 28,57 & 0,3 & 0,59 & 63,16 & 10,91 & 18,61 \\
& Werther & 28,21 & 1,67 & 3,15 & 69,8 & 11,52 & 19,78 \\
\multirow{2}{*}{ ORG } & Bundestagsdebatten & 3,7 & 0,32 & 0,59 & 42,28 & 37,1 & 39,52 \\
& & & & & & & \\
\multirow{2}{*}{ WRK } & Bundestagsdebatten & 0 & 0 & 0 & 42,86 & 4,05 & 7,25 \\
& Werther & 0 & 1,35 & 0 & 0 & 0 & 0 \\
\multirow{2}{*}{ CNC } & Adorno & 0 & 0 & 0 & 60,66 & 45,73 & 52,18 \\
\hline
\end{tabular}

CRETA-Projekts ergeben sich IAA-Werte zwischen 0,66 und 0,89 (vgl. Tabelle 2), wobei die Berechnung die zeitliche Dimension des Annotationsprozesses unberücksichtigt lässt und diesbezüglich davon auszugehen ist, dass die anfängliche Übereinstimmung geringer ausfiel und der Wert nach Schärfung der Richtlinien und Training der Annotierenden anstiegen ist.

Die Sichtung der nicht-übereinstimmenden Annotationen zeigt, dass inkongruente Annotationen dreierlei Ursachen haben können: Erstens können Unstimmigkeiten darüber herrschen, ob es sich bei einem Ausdruck um eine Entitätenreferenz handelt oder nicht; zweitens können die Annotierenden zwar den gleichen Ausdruck annotiert, dabei aber verschiedene Entitätenklassen ausgewählt haben (was insbesondere metonymische Verwendungsweisen betrifft); drittens können Inkongruenzen darauf zurückgehen, dass die Grenzen eines Referenzausdrucks nicht übereinstimmend annotiert wurden. Die iterative Überarbeitung der Richtlinien kann generell nur diejenigen Inkongruenzen beseitigen, die auf unzureichend geschärften Definitionen oder auf unterschiedlichen Vorverständnissen seitens der Annotierenden zurückzuführen sind (vgl. Gius und Jacke 2017).

Wie in Abschnitt 2.2 beschrieben, werden auf Grundlage der manuell annotierten Daten korpusspezifische Entitätenreferenztagger trainiert. Diese werden 
im Vergleich zu einer baseline evaluiert. ${ }^{8}$ Die baseline repräsentiert den Status quo für eine Aufgabe und bildet damit einen Ausgangspunkt für die Evaluation neuer Modelle. Für die Aufgabe der Entitätenreferenzerkennung wurde als baseline ein Modell des Stanford Named Entity Recognizer (Finkel et al. 2005) für das Deutsche verwendet (Faruqui und Padó 2010). Tabelle 3 fasst die Evaluation der Modelle zusammen. Der precision-Wert steht für die Genauigkeit eines Systems und gibt an, wie viele der vorhergesagten Annotationen korrekt sind, der recall misst die Vollständigkeit, indem er angibt, wie viele der zu identifizierenden Objekte von einem System gefunden worden sind (vgl. Manning und Schütze 1999, S. 267-269). Im $\mathrm{F}_{1}$-score werden die Werte precision und recall zusammengefasst (harmonisches Mittel, vgl. Manning und Schütze 1999, S. 269). Als korrekt (true positive) wurden in dieser Evaluation nur die vorhergesagten Referenzen gezählt, die mit den Referenzdaten exakt übereinstimmen und z. B. auch anhängende Relativsätze korrekt erkennen.

Der in CRETA verwendete Entitätenreferenzerkenner basiert auf dem Verfahren conditional random fields (Lafferty et al. 2001), bei dem Kontextabhängigkeiten zwischen den Entscheidungen für einzelne Wörter mit berücksichtigt werden. Im Gegensatz zu einer einfachen Klassifikation, bei der individuelle Wörter unabhängig von den Entscheidungen im Kontext klassifiziert würden, kann das Modell erlernen, dass ein Wort eher Teil einer Entitätenreferenz ist, wenn davor z. B. ein definiter Artikel steht. Die verwendeten Merkmale sind die Wortform, die (automatisch erkannte) Wortart, eine Angabe darüber, ob die Wortform groß- und kleingeschrieben im Korpus vorkommt, eine Namensliste (die korpusspezifische häufige Namen enthält), sowie eine Charakterisierung der Buchstabenmuster, die im Wort vorkommen. ${ }^{9}$ Für die technische Umsetzung wurde die Bibliothek clearTk ${ }^{10}$ (Bethard et al. 2014) verwendet, die ihrerseits das Tool mallet ${ }^{11}$ (McCallum 2002) einbindet. Trainiert und getestet wurde mit fünffacher Kreuzvalidierung.

Die Evaluation zeigt, dass die korpusspezifisch trainierten Entitätenreferenztagger insgesamt deutlich bessere Ergebnisse erzielen können als das state of the

\footnotetext{
8 Die Evaluation des Entitätenreferenztaggers erfolgte im Rahmen des Workshops „CUTE: CRETA Unshared Task zu Entitätenreferenzen“ bei der DHd 2017 (Reiter, Blessing et al. 2017). Das annotierte Korpus steht über folgenden Link zum Download zur Verfügung: https://www.creta.unistuttgart.de/cute/datenmaterial/.

9 Dabei wird jedes Zeichen des Wortes ersetzt durch seine Unicode-Kategorie (z. B. das Zeichen „a“ durch ,Letter lowercase‘, Ll). Danach werden nachfolgend gleiche Kategorien zusammengefasst. Aus der Zeichenfolge „Parzival“ wird also ,LuLl‘.

10 http://cleartk.github.io/cleartk/

$11 \mathrm{http}: / /$ mallet.cs.umass.edu
} 
art NER-Modell. ${ }^{12}$ Die stark variierenden Ergebnisse für die verschiedenen Textkorpora sind $\mathrm{u}$. a. dem unterschiedlich großen Umfang der Trainingsdaten (Tabelle 1) und der Verteilung der Entitätenklassen geschuldet. Die besten Ergebnisse werden bei einem häufigen Vorkommen einer Entitätenklasse im Trainingskorpus erzielt, beispielsweise bei Figuren (PER) in den mittelhochdeutschen Artusromanen oder bei Organisationen (ORG) in den Bundestagsdebatten. Insgesamt fällt auf, dass die precision des Entitätenreferenztaggers deutlich höher ist als der recall. Das bedeutet, dass der Tagger zwar gefundene Objekte korrekt klassifiziert, er aber die Mehrheit der relevanten Objekte nicht findet. Vor dem Hintergrund, einen zeitsparenden semi-automatischen Annotationsprozess anzustoßen, ist es sinnvoll, den recall-Wert zu verbessern (ggf. auch zulasten der precision), also möglichst viele Objekte in einem Text zu finden.

Für das mittelhochdeutsche Romankorpus wird unter Hinzunahme weiterer Features die Entwicklung des Entitätenreferenztaggers fortgeführt (Blessing et al. 2017). Durch die Implementierung eines mittelhochdeutschen Wortartenerkenners (Echelmeyer et al. 2017) ${ }^{13}$, von Namenslisten und eines Features zur Groß-/Kleinschreibung wird eine deutliche Verbesserung der automatischen Vorhersagen erzielt. Des Weiteren werden verschiedene Evaluationssettings getestet und miteinander vergichen: Während im oben verwendeten engen Evaluationssetting eine Annotation nur dann als korrekt bewertet wird, wenn sowohl die Entitätenklasse (PER, LOC, ORG, WRK, CNC) als auch die Grenzen des Referenzausdrucks exakt übereinstimmen, gilt im weiten Evaluationssetting eine Annotation als korrekt, sobald die Entitätenklasse stimmt und sie mindestens ein Token Überlappung mit der Goldannotation aufweist.

Die Ergebnisse in Tabelle 4 zeigen, dass zum einen die Implementierung zusätzlicher Features zu erheblichen Verbesserungen der Entitätenreferenzerkennung führt und dass zum anderen viele Fehler auf inkorrekte Grenzen des Referenzausdrucks zurückgehen, was über das weite Evaluationssetting aufgefangen wird. Für das Untersuchungsvorhaben, die Vorkommen von Entitäten aus ei-

12 Anzumerken ist, dass das verwendete Stanford NER-Modell nur die Klassen PER, LOG und ORG umfasst und daher bei den Klassen WRK und CNC keine Entitäten findet. Des Weiteren ist er für eine Eigennamenerkennung entwickelt, so dass Entitätenreferenzen über Gattungsnamen nicht erfasst werden.

13 Unser part-of-speech-Modell für das Mittelhochdeutsche kann von der Webseite des Instituts für Maschinelle Sprachverarbeitung heruntergeladen werden: https://www.ims.uni-stuttgart. de/forschung/ressourcen/werkzeuge/pos_tag_mhg/; es ist auch im CLARIN Virtual Language Observatory (https://vlo.clarin.eu), einer Suchmaschine für linguistische Ressourcen und Werkzeuge, nachhaltig auffindbar. Eine Online-Version des Taggers steht zur Verfügung unter: http: //clarin05.ims.uni-stuttgart.de/mhdtt/index.html. 


\begin{tabular}{lccc}
\hline Evaluation & precision & recall & $\mathbf{F}_{1}$ \\
\hline eng & 73,94 & 54,79 & 62,94 \\
weit & 94,85 & 73,82 & 83,02 \\
\hline
\end{tabular}

Tab. 4: Evaluation des weiterentwickelten Entitätenreferenztaggers (ERT2). Getestet wurden PER-Referenzen auf Parzival.

nem Text zu extrahieren, scheint generell die weite Evaluation geeigneter zu sein, da - sofern eine Entitätenreferenz erfasst wurde - die exakten Grenzen des Ausdrucks vernachlässigbar sind. Für semantisch motivierte Untersuchungen kann hingegen die enge Evaluation, die die Erfassung der gesamten Nominalphrase evaluiert, sinnvoller sein. Prinzipiell sollte die Evaluation maschineller Verfahren immer vor dem Hintergrund des spezifischen Forschungsinteresses erfolgen und entsprechend reflektiert werden.

\section{Multidisziplinäre Zugänge zu Entitäten in den Geistes- und Sozialwissenschaften}

\subsection{Sozialwissenschaftlicher Zugang}

Politische Parteien, internationale Organisationen oder Institutionen sind seit jeher zentrale Analyseobjekte der empirischen sozialwissenschaftlichen Forschung. Sie werden spätestens seit dem linguistic turn (Rorty 1992) in den Sozialwissenschaften auch mehr und mehr mittels textanalytischer Methoden in zunächst kleinen und zunehmend größeren Mengen von Textdokumenten untersucht, beispielsweise in Parteiprogrammen, offiziellen Regierungsdokumenten oder Zeitungstexten. Innerhalb transnationaler europäischer Mediendebatten wird darüber hinaus die Sichtbarkeit und die semantische Bewertung verschiedener politischer Entitäten (z. B. der Europäischen Union) untersucht (z. B. Kantner 2015).

Sprachliche Bezüge auf unterschiedliche Entitätentypen sind also Bestandteil zahlreicher sozialwissenschaftlicher Forschungsinteressen und Fragestellungen. In unserem Projekt haben wir die Nennung verschiedener Entitätentypen als Bestandteil multipler kollektiver Identitäten im politischen Diskurs untersucht. Kollektive Identität verstehen wir als gemeinsames normatives Selbstverständnis, das von den Mitgliedern einer Wir-Gemeinschaft geteilt wird (Tietz 2002; Kantner 2004). In modernen Gesellschaften sind Individuen in ein komplexes Geflecht multipler Zugehörigkeiten eingebunden, die je nach sozialem oder politischem Kontext unterschiedliche Bedeutung gewinnen können (Kantner und Tietz 2013). Über den sprachlichen Bezug auf bestimmte Entitäten können Sprecher`innen 
hierbei ihre Identifikation mit einem bestimmten Kollektiv zum Ausdruck bringen. Zwar sind nicht alle sprachlichen Bezüge auf Entitäten automatisch auch Äußerungen von kollektiver Zugehörigkeit, doch setzt die Äußerung kollektiver Identifikation oftmals einen sprachlichen Bezug auf Entitäten voraus. Im Sinne eines mehrstufigen Analyseprozesses, der sich der semantischen Komplexität des Konzepts kollektiver Identität schrittweise annähert, bildet die (semi-automatische) Annotation von Entitätenreferenzen daher eine sinnvolle Möglichkeit, große Textkorpora auf die für die Fragestellung relevanten Texte und Textpassagen einzugrenzen.

Wir haben für unsere Analyse das PolMine ${ }^{14}$-Korpus verwendet(Blätte und Blessing 2018). Hierbei handelt es sich um ein Textkorpus, das die Plenarprotokolle des Deutschen Bundestags im Zeitraum von 1996 bis 2016 enthält. Die Auswahl dieses Textmaterials ermöglichte es uns, Referenzen auf politische Entitäten innerhalb alltagssprachlicher Debatten zu analysieren. Um Trainingsdaten für die semi-automatische Annotation zu generieren, annotierten wir sämtliche Nennungen politischer Entitäten auf einer Teilmenge der transkribierten Plenarprotokolle. Annotiert wurden Referenzen auf Personen (PER), Organisationen (ORG), Orte (LOC) und Werke (WRK). Bei der Anpassung der universellen Annotationsrichtlinien an das ausgewählte Textmaterial haben wir weitere, ergänzende Zusatzrichtlinien erstellt. Dies erleichterte die Identifikation relevanter Textstellen und erhöhte insgesamt die Einheitlichkeit der Annotationsergebnisse.

Für die verschiedenen Klassen der fokussierten Entitäten enthielten die Zusatz-Guidelines allgemeine Definitionen und Beispiele, wie in konkreten Sätzen oder Passagen auf die verschiedenen Entitäten referiert werden kann. Organisationen wurden beispielsweise anhand von Referenzen wie „die Europäische Union“, „die deutsche Bundesregierung“ benannt. Auf Personen wurden über Ausdrücke wie „Frau Merkel“ oder „die deutsche Bundeskanzlerin“ referiert. Referenzen auf Orte fanden sich, oftmals gemeinsam mit lokalen Präpositionen, in Nennungen wie „Paris“, „Berlin“ oder „Brüssel“. Auf Werke wurde in Ausdrücken wie z. B. „die Kopenhagener Kriterien“ referiert.

(1) a. Die Deutschen zwingen den Griechen einen rigiden Sparkurs auf.

b. Berlin muss sich seiner internationalen Verantwortung stellen.

Probleme, die bei der Annotation auftraten, betrafen oftmals die Disambiguierung mehrdeutiger Textstellen. Organisationen wurden z. B. häufig über Referenzen auf konkrete Orte wie „Berlin“, „Brüssel“ oder „New York“ adressiert. Auch

14 Für weitere Informationen zum PolMine Projekt, siehe https://polmine.github.io/ 
Nennungen von Personen oder Gattungen von Personen referierten inhaltlich in manchen Fällen auf Organisationen, z. B. wenn „die Deutschen“ „den Griechen“ „einen rigiden Sparkurs“ aufzwangen (1a), war eigentlich eine Organisation gemeint (in diesem Fall die Bundesregierung). In vielen Fällen ließen sich solche Mehrdeutigkeiten über den jeweiligen Satzkontext disambiguieren. Organisationen wurden oftmals per Städte- oder Ländernamen adressiert, wenn diese mit Modalverben wie „muss“, „,sollte“ oder „kann“ kookkurrierten (1b). Auf Orte wurde hingegen öfter über lokale Präpositionen wie „in“, „,nach“ oder „aus“ referiert. Um die Einheitlichkeit der Annotationen zu erhöhen, wurden Entscheidungshilfen für rekurrierende ambivalente Fälle in die Zusatz-Guidelines aufgenommen.

In anderen Fällen kam es zu voneinander abweichenden Annotationen ein und derselben Textstelle, die auch im Rahmen der Adjudikation (Zusammenführung und Auflösung der Annotationsunterschiede) nur schwer aufzulösen waren. Im vorherigen Beispiel der ,Deutschen‘ und ,Griechen“ war es tatsächlich schwierig, einheitlich zu bestimmen, ob nun dem griechischen Staat (ORG) oder vielmehr den Griechen als Gruppe von Personen (PER) ein rigider Sparkurs aufgezwungen wird. Neben der inhärenten Multireferentialität einzelner Terme, war die ,Bestimmtheit‘ von Gattungsreferenzen eine weitere Quelle für abweichende Annotationen. Ob mit ,den Deutschen“, die eine ,historische Verantwortung haben' eine bestimmte oder eine unbestimmte Gruppe von Personen gemeint ist, ist eine Interpretationsfrage. Um die Einheitlichkeit der Annotation zu erhöhen, wurden bei solch mehrdeutigen Textstellen möglichst generische Entscheidungen getroffen, die anschließend als ergänzende Guidelines in die Annotationsrichtlinien aufgenommen wurden.

\subsection{Mediävistischer Zugang}

Die mediävistische Fachgruppe untersucht Figuren und ihre Relationen in einem Korpus mittelhochdeutscher Artusromane (Erec und Iwein Hartmanns von Aue, Parzival Wolframs von Eschenbach) ${ }^{15}$ (Ketschik in Vorbereitung). Figuren sind, allgemein gesprochen, ,mit ihrer sinnkonstitutiven und handlungsprogressiven Funktion ein[] elementare[r] Baustein der fiktiven Welt“ (Platz-Waury 1997a, S. 587) und daher für viele Forschungsfragen grundlegend. In mediävistischen Kontexten wird vor allem die charakteristische Figurenzeichnung als „flache“ Fi-

15 Es werden folgende Editionen verwendet: Hartmann von Aue 1963, Hartmann von Aue 1968 und Wolfram von Eschenbach 1891. 
guren $^{16}$ (Forster 1949, S. 77) oder „Handlungsträger“ (Propp 1972) diskutiert, derzufolge den Figuren nur eine „Minimalausstattung“ (Haferland 2013, S. 105-114) an Individualität und Innerlichkeit zugesprochen wird. Stattdessen werden Figuren stärker über ihr Handeln definiert, was mit der strukturalistischen Figurenkonzeption einhergeht, die ihre Funktionsgebundenheit betont (vgl. Schulz 2012, S. 12).

In den ausgewählten mittelhochdeutschen Artusromanen sollen die Figuren vor allem hinsichtlich ihrer Zentralität, ihrer Relationen und ihrer Bedeutung für die Handlung untersucht werden. Hintergrund ist ein Vergleich zwischen den Artusromanen Hartmanns von Aue (Erec und Iwein) und dem deutlich komplexeren Artusroman Wolframs von Eschenbach (Parzival). Mittels datenbasierter Verfahren und unter Rückgriff auf die Methode der sozialen Netzwerkanalyse ${ }^{17}$ wird das Figureninventar der Erzählungen analysiert und ausgewertet (vgl. Braun und Ketschik 2019). Dabei soll eine dynamische Visualisierung der Netzwerke ermöglichen, die Figurenvorkommen und -relationen über den Verlauf der Handlung hinweg nachzuvollziehen. Auch der sukzessive Aufbau des Figureninventars und mögliche Veränderung in den Positionen einzelner Knoten über die Erzählzeit hinweg werden auf diese Weise sichtbar. Ergänzend werden netzwerkanalytische Metriken herangezogen, die u.a. Auskunft über die Größe und Verbundenheit der Netzwerke und die strukturelle Relevanz der Akteure geben, so dass die ausgewählten Texte quantitativ miteinander verglichen werden können.

Weiterführend soll auch das Zusammenspiel von Figuren und Räumen in den Blick genommen werden (vgl. hierzu auch Viehhauser 2020 ab Seite 373 in diesem Band). Der Handlungsraum ist in der mittelhochdeutschen Epik meist dichotom strukturiert und semantisiert (Lotman 1972) - so steht im höfischen Roman der kultivierte höfische Raum dem gefährlichen, unkultivierten Abenteuerraum konträr gegenüber. In den ausgewählten Artusromanen sollen die Räume zum einen in Bezug auf ihre handlungsstrukturierende Funktion und zum anderen bezüglich möglicher Wechselwirkungen mit der Kategorie der Figur untersucht werden. Hierbei interessiert, welche Figuren bestimmten semantischen Räumen fest zugeordnet sind, und welche Figuren in der Lage sind, die Raumgrenzen zu überschreiten. ${ }^{18}$

16 ,Flache‘ Figuren verkörpern - im Gegensatz zu ,runden` Figuren - nur ,eine einzige Idee oder Eigenschaft‘. Neutralere Begriffe als ,flach` und ,rund‘ führen Lahn/Meister ein, die stattdessen von „einfachen“ und „komplexen“ Figuren sprechen (Lahn und Meister 2016, S. 239).

17 Zur sozialen Netzwerkanalyse vgl. allgemein Jansen (2003) sowie stärker literaturwissenschaftlich Trilcke (2013).

18 Ein wesentliches Strukturmerkmal von Räumen in Texten ist Lotman zufolge ihre unüberschreitbare Grenze, die sich nur für den Helden als überwindbar erweist (Lotman 1972, S. 341). 
Zur Durchführung der Analysen ist es notwendig, die Vorkommen der Figuren sowie die Handlungsräume im Text adäquat zu erfassen, was über die Annotation der Entitätenreferenzen der Klassen PER und LOC erfolgt.

Für das Vorhaben, Figurenvorkommen und -relationen im Korpus ausgewählter mittelhochdeutscher Artusromane zu untersuchen, ist die Erfassung von Figurenreferenzausdrücken grundlegend. Nach Umsetzung des Untersuchungsvorhabens auf die oben beschriebene Weise können folgende Ergebnisse, aber auch Herausforderungen und Grenzen herausgestellt werden. Zunächst ist festzuhalten, dass die Erfassung von Figurenreferenzausdrücken (via Eigen- und Gattungsname) für die angestrebten Analysen grundlegend ist und aus den folgenden Gründen geeignet erscheint: Die Figurenvorkommen lediglich über Eigennamen zu erfassen, führt nach eingehender Analyse der Daten zu verzerrenden Ergebnissen, da die Figuren zum einen deutlich häufiger über Gattungsnamen erwähnt werden als über Eigennamen, und da zum anderen die Verteilung der Eigen- und Gattungsnamen je nach Figur unterschiedlich ausfällt. So werden die Protagonisten in der Regel häufig mit Gattungsnamen bezeichnet („der ritter“, „der helt“), während auf andere Figuren häufiger mit ihrem Eigennamen referiert wird. Die Berücksichtigung der Gattungsnamen, die mit den disziplinübergreifenden Richtlinien korreliert, ist für das mittelhochdeutsche Textkorpus somit essenziell. Dieser Umstand macht allerdings zur weiteren Nutzung der Daten einen Folgeschritt notwendig, der darin besteht, die Ausdrücke auf die Figur, auf die sie referieren, aufzulösen (entity grounding). Nur durch diesen zusätzlichen Schritt kann die notwendige Information, welche Figureninstanz genannt wird, mit der textuellen Oberflächenform verknüpft werden. Da ein Großteil der Appellativbezeichnungen mehrdeutig ist (,die vrouwe“ kann je nach Textstelle auf verschiedene Figuren referieren), muss die Auflösung der Begriffe unter Berücksichtigung des jeweiligen Kontextes vorgenommen werden, was trotz technischer Unterstützung einen hohen Zeitaufwand erfordert.

Ferner ist anzumerken, dass - anders als die generischen Richtlinien es vorsehen - die Erfassung der maximalen Nominalphrase für die weiteren Analysen nicht notwendig ist. Um zu extrahieren, wann welche Figur im Text genannt wird, ist es lediglich wichtig, einen Teil des Referenzausdrucks (wenn möglich den syntaktischen Kopf) zu erfassen - vor- oder nachgestellte Adjektive, Determinanten, Appositionen oder andere Ergänzungen sind hingegen vernachlässigbar. Die Richtlinien wurden im Laufe des Annotationsprozesses also dahingehend angepasst, dass auch eine unvollständige Entitätenreferenz als ausreichend betrachtet wird. Lediglich bei verschachtelten Referenzausdrücken blieb es wichtig, die Grenzen korrekt zu erfassen, um später die eingebetteten Ausdrücke identifizieren $\mathrm{zu}$ können. 
Da im Rahmen des Untersuchungsvorhabens große Textmengen zu bewältigen waren, wurde nach der anfänglichen manuellen Annotation ein maschinelles Lernverfahren angestoßen, um einen Entitätenreferenzerkenner für mittelhochdeutsche Texte zu trainieren (vgl. Abschnitt 2.2). Mithilfe des Entitätenreferenztaggers konnte der Annotationsprozess erheblich zeiteffizienter gestaltet werden, da sich die manuelle Arbeit im Anschluss darauf beschränkt, die Vorhersagen zu kontrollieren und eventuelle fehlende Referenzausdrücke nachzuannotieren.

Eine Herausforderung der (semi-)automatischen Annotation bestand in der Adaption des Modells an neue Texte, in denen andere Eigennamen und Appellative vorkommen oder ein anderer (autor-/gattungsspezifischer) Schreibstil vorliegt als in den Daten, mit denen ein Modell trainiert wurde. Um die Differenz zwischen Quell- und Zieldomäne zu überbrücken, wurde - sobald der Entitätenreferenzerkenner auf einen neuen Roman angewandt wurde - je ein Teil des Zieltextes von Grund auf annotiert und in die Trainingsdaten inkludiert. Dadurch musste zwar zunächst mehr manuelle Arbeit investiert werden, die manuelle Mehrarbeit konnte aber dem Absinken der Erkennungsrate entgegenwirken und somit den Annotationsprozess des gesamten Textes effizienter gestalten.

Auch der Schritt des entity grounding wurde semi-automatisch unterstützt, indem Vorhersagen darüber generiert wurden, auf welche Figur ein bestimmter Ausdruck an einer bestimmten Stelle referiert. Über eine spezielle Benutzeroberfläche im Annotationstool CRETAnno wurden sowohl der annotierte Referenzausdruck mit einem Kontextfenster als auch die sechs wahrscheinlichsten Figureninstanzen angezeigt, so dass aus dieser Liste nur noch die korrekte Instanz ausgewählt oder - sofern nicht enthalten - über eine zuschaltbare Figurenliste herausgesucht werden musste. Trotz der technischen Unterstützung handelte es sich um einen aufwändigen, für das Forschungsinteresse aber unverzichtbaren Arbeitsschritt.

Generell ist festzuhalten, dass die Umsetzung des angestrebten Untersuchungsvorhabens deutlich komplexer ist, als zuvor angedacht. Die Erfassung der Referenzausdrücke ist für das Vorhaben zwar essenziell, es ist aber eine Vielzahl weiterer Schritte notwendig. So muss neben dem bereits skizzierten entity grounding auch der Status der Entitäten berücksichtigt werden, um Figuren der fiktiven Welt von anderen Figuren oder Personen (z. B. von historischen Personen) zu unterscheiden. Ferner ist zu differenzieren, ob eine Entitätenreferenz in Figurenrede steht oder nicht, wofür alle Passagen direkter Rede identifiziert werden müssen. Und zuletzt müssen eingebettete Referenzausdrücke erkannt und herausgefiltert werden, da sie in der Regel nicht auf Figuren referieren, die an der entsprechenden Stelle des Textes agieren. Somit ist für eine adäquate Umsetzung des Untersuchungsvorhabens die reine Erfassung der Entitätenreferenzen nicht ausreichend. Die Integration der weiteren Schritte führte dazu, das ein komple- 


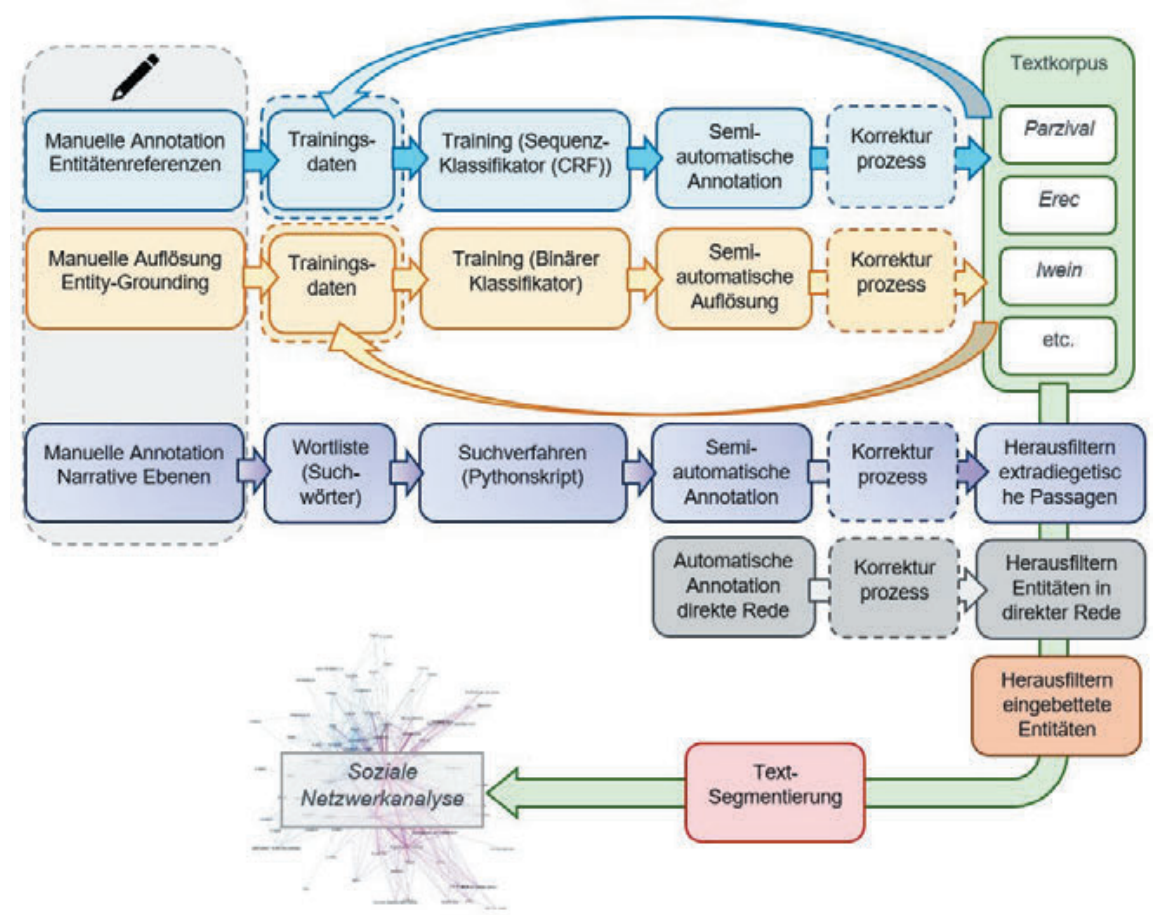

Abb. 6: Gesamtworkflow zur Sozialen Netzwerkanalyse mittelhochdeutscher Artusromane

xer Workflow entstand, der über den gemeinsamen methodischen Ansatz weit hinausgeht (vgl. Abbildung 6 und Ketschik in Vorbereitung).

Eine weitere Herausforderung bestand in der Annotation der Orte (LOC), da das Konzept des Ortes oder Handlungsraums in fiktionalen Texten unscharf definiert ist und weitere Unterscheidungskriterien, etwa ,Ereignisregion' und ,erwähnte räumliche Gegebenheit', benötigt werden (vgl. Barth und Viehhauser 2017 und Viehhauser 2020, ab Seite 373 in diesem Band). Zusätzliche Schwierigkeiten bestanden darin festzulegen, wie zum einen mit der Granularität von Räumen oder Raumobjekten umgegangen werden soll (der Ort Nantes ist eindeutig ein Handlungsraum, aber wie sieht es mit einem Zimmer in der Burg von Nantes oder mit einem Bett im Burgzimmer aus?) und wie zum anderen Objekte zu kategorisieren sind, die zur räumlichen Verortung herangezogen werden (ist z.B. ein Stuhl als Ortsreferenz zu zählen, wenn eine Figur darauf sitzt?). Und zuletzt kann in fiktionalen Texten potenziell jedes Objekt zum Raum werden (man denke an einen Flaschengeist, der die Flasche zum Lebensraum hat), so dass jeweils kontextspezifisch über den Status eines Objekts entschieden werden musste. Diese 
Herausforderungen machten es notwendig, text- und korpusspezifische Richtlinien für den Umgang mit Raumobjekten einzuführen.

Für die folgenden Analysen von Figuren- und Raumkonstellationen wurde ferner festgestellt, dass die annotierten Raumausdrücke nicht zwangsweise Aufschluss über den Schauplatz der Handlung geben (wurde beispielsweise eine ,Kemenate‘ annotiert, fehlte die Information, wo sich dieses Schlafgemach befindet). Für die Erfassung der Handlungsräume erwies es sich daher als geeigneter, eine Segmentierung des Textes in Raumsegmente vorzunehmen, bei der ganze Handlungsabschnitte einem bestimmten Raum zugeordnet wurden. Auf diese Weise konnte für die anschließenden Analysen die Information extrahiert werden, welche Figuren in welchen Handlungsräumen agieren.

\subsection{Zugang der Neueren Deutschen Literatur}

Für die Neuere Deutsche Literatur steht die Untersuchung und Charakterisierung von Figuren und deren Konstellation im Fokus, allen voran der Dreiecksbeziehung, die sich in Johann Wolfgang von Goethes maßgebendem Briefroman Die Leiden des jungen Werthers manifestiert und zu einem Bezugspunkt in der literarischen Rezeption des Werks wird.

Die Adaptationen, die in Referenz auf Goethes Briefroman entstanden sind, orientieren sich in unterschiedlicher Hinsicht am Originalwerk. So wurde der Werther seit der Veröffentlichung seiner ersten Fassung 1774 in verschiedenen Gattungen literarisch und kritisch verarbeitet, satirisch verfremdet, parodiert oder schlicht nachgeahmt. Insgesamt konnten bisher rund 140 deutsche Werke identifiziert werden, ${ }^{19}$ (vgl. hierzu Appell 1882; Atkins 1949; Scherpe 1970; Richter 2017) die als ,Wertheriaden' gelten. ${ }^{20}$ Die Bezüge auf das Originalwerk fallen in den Adaptationen unterschiedlich stark aus und und können sowohl formaler, struktureller als auch inhaltlicher Art sein. (Martens 1985; Horré 1997). Die im Rahmen von CRETA vorgenommenen Analysen konzentrieren sich auf die

19 Goethes Werther erfuhr auch eine starke internationale Rezeption. 1776 wurde der Briefroman bereits ins Französische und 1779 ins Englische übersetzt. Zu Lebzeiten Goethes erschienen zudem Übersetzungen ins Russische, Italienische, Niederländische, Schwedische, Spanische, Dänische und Portugiesische (vgl. Goedeke und Goetze 1979). Es lassen sich auch fremdsprachige Wertheriaden nachweisen, wie unter anderem die 1785 anonym veröffentlichte englischsprachige Adaptation Eleonora. From the Sorrows of Werther. A Tale, in dem Werther und Lotte ihre Rollen tauschen, vgl. hierzu u.a. Richter 2017.

20 Die Anzahl der deutschen Wertheriaden setzt sich aus 33 Dramentexten, 29 Gedichten und 78 Prosatexten zusammen. Von den 78 Prosatexten werden in unserer Analyse 30 Texte, darunter Brief- und Tagebuchromane, Novellen und Erzählungen berücksichtigt. 
Gattung der Prosatexte. Der Zugang zu diesem heterogenen Textkorpus erfolgt über die Entitätenklassen Figuren/Personen (PER), Orte (LOC) und (literarische) Werke (WRK). Die Annotation der Entitätenreferenzen beschränkt sich zunächst auf das Originalwerk, mit dem Vorhaben, die Annotation auf die überarbeitete Fassung des Werthers von 1787 sowie weitere Wertheriaden auszuweiten, um auch hierin Figuren, Orte und intertextuelle Verweise $\mathrm{zu}$ erfassen und so eine Vergleichbarkeit der Werke $\mathrm{zu}$ ermöglichen. Vorrangiges Ziel ist die Erfassung von Figurenkonstellationen sowie die Untersuchung und die Beantwortung der Frage, in welchen Wertheriaden sich die charakteristische Dreiecksbeziehung ebenfalls identifizieren lässt. Zur Analyse werden Netzwerke des Originaltexts und der Werther-Adaptationen generiert.

Die Analyse der Figurenkonstellationen in literarisch-fiktionalen Texten ermöglicht es, die dynamische Struktur der Interaktion zwischen den Figuren zu erfassen. Die jeweiligen Konstellationen übernehmen dabei nicht nur eine „handlungsgestaltende Funktion“, die Figuren werden durch die „Kontrast- und Korrespondenzrelationen“ (Platz-Waury 1997b, S. 591) zudem auch strukturiert und charakterisiert (Pfister 2001, S. 232-235). Dadurch bilden bestimmte Figurenkonstellationen die Grundlage für Konfliktstrukturen, etwa die Gegenüberstellung eines Protagonisten mit einem Antagonisten. In Goethes Werther ist diese ,klassische' Konstellation um eine weitere Figur, Lotte, erweitert. Diese bildet neben dem emotionalen Protagonisten Werther, der sich in sie verliebt, und ihrem Verlobten Albert, der den Widersacher Werthers darstellt, eine Grenzgängerfigur. Viele Wertheriaden greifen diese tragische Liebesgeschichte auf und stellen sie in den Fokus, um eine deutliche Referenz auf den Originaltext zu erzeugen.

Der komplexe Aufbau von Goethes Briefroman birgt Herausforderungen für die Annotation der Entitätenklassen, insbesondere der Figuren (PER), und hat dadurch in unterschiedlicher Hinsicht zur Schärfung dieser Klasse beigetragen.

Der Roman beginnt mit den mahnenden Worten des fiktiven Herausgebers:

Was ich von der Geschichte des armen Werther nur habe auffinden können, habe ich mit Fleiß gesammelt [...] Und du gute Seele, die du eben den Drang fühlst wie er, schöpfe Trost aus seinem Leiden, und laß das Büchlein deinen Freund sein, wenn du aus Geschick oder eigener Schuld keinen nähern finden kannst. (Goethe 1899, S. 4)

Diese Worte dienen nicht nur der Rahmung der folgenden Briefe Werthers, sondern werfen auch erste Fragen an die Richtlinien auf: Werden Ansprachen an fiktive Adressaten (,gute Seele“) als PER annotiert?

In Hinblick auf den Status der Entitätenreferenz wird in den CRETA-Richtlinien zunächst nicht unterschieden, ob solche Ansprachen innerhalb der Figurenrede, einer Protokollanmerkung, der Exegesis, Diegesis oder Metadiegesis erwähnt wer- 
den. Eine entsprechende Bestimmung wird erst nach Abschluss der Vollannotation und im Kontext der darauf aufbauenden Analyse getroffen. Für die Annotation ist jedoch entscheidend, ob sich aus dem Zusammenhang entscheiden lässt, ob sich eine Entitätenreferenz auf eine der Figuren im Text bezieht oder nicht. Mit der „gute[n] Seele“21 (vgl. Lahn und Meister 2016, S. 17) wird keine der Figuren im Text adressiert, weshalb diese Ansprache folglich unannotiert bleibt.

Die Diegese im Roman bilden vorrangig 81 Briefe, die Werther an seinen Freund Wilhelm richtet und in denen er von seinem abgeschiedenen Leben in Walheim und seiner aufkeimenden Liebe zur Amtstochter Lotte berichtet. Antwortbriefe seines Briefpartners sind nicht enthalten, wodurch eine autodiegetische Erzählweise und Monoperspektive auf die Geschehnisse entsteht.

Der Herausgeber führt die Geschichte Werthers zu Ende, nachdem sich dieser durch einen Schuss aus der Waffe seines Gegenspielers Albert das Leben genommen hat. Dieser Aufbau macht sich bei der Annotation der Figuren dahingehend bemerkbar, dass ,Werther' nur selten erwähnt wird, da er sich als Erzähler in seinen Briefen nicht selbst beim Namen nennt. Eine Nennung häuft sich erst in den abschließenden Passagen aus dem Teil „Der Herausgeber an den Leser“ oder findet sich in den indirekt wiedergegebenen oder zitierten Aussagen anderer Figuren. Für die Generierung eines Netzwerkes und die Identifikation der charakteristischen Dreiecksbeziehung hat dies zur Folge, dass als heuristischer Ansatz zunächst alle Vorkommen des Pronomens ,Ich' mit Werther synonym gesetzt werden müssen (Barth und Murr 2017). Eine exakte Zuordnung des Pronomens ermöglicht erst eine Auflösung der Koreferenzen.

Bei der Generierung des Netzwerkes gilt es zudem zu berücksichtigen, dass manchen Figuren im Werther ein anderer Status zukommt, da sie einer unterschiedlichen fiktiven Welt angehören. Denn es sind nicht nur die Naturbeschreibungen, die das Seelenleben des Protagonisten widerspiegeln, sondern auch die intertextuellen Verweise (WRK). So liest Werther, wenn es ihm gut geht, in seinem Homer, erkennt in dem gemeinsamen Ausruf „Klopstock“ am Ball die scheinbare Seelenverwandtschaft zu Lotte und seine Ausweglosigkeit nach seinem Selbstmord wird durch das aufgeschlagene Drama Lessings Emilia Galotti verdeutlicht. Bevor Werther diesen drastischen Schritt wagt, liest er am Vorweihnachtsabend Lotte aus den Gesängen Ossians vor. Diese insgesamt drei Passagen (Colma, Ryno und Alpin) sind gespickt mit Figurennennungen, deren Häufung auch in der Visualisierung und dem Vergleich der (unterschiedlichen) Annotationen deutlich

21 Bei der „gute[n] Seele“, die vom Herausgeber angesprochen wird, handelt es sich um den „,Hörer‘ [bzw. hier Leser] der herausgegebenen Briefe und der nachgeordneten Erzählung des fiktiven Herausgebers, an den dieser sich direkt wendet.“ 
hervortritt (vgl. hierzu auch den Beitrag von Baumann et al. (2020) ab Seite 270 in diesem Band). Bei der Analyse des Netzwerkes des gesamten Textes gilt es somit, eine Unterscheidung von aktiv präsenten Figuren der Diegese und Figuren aus anderen fiktiven Welten zu treffen. Bezüglich der Annotation der aktiv präsenten Figuren kommt hinzu, dass der Erzähler Werther in seinen Briefen nicht alle Figuren ausschließlich bei ihrem Namen nennt, sondern in diesen Fällen u. a. zu Kosenamen übergeht. Umso stärker sich beispielsweise das Verhältnis von Werther und Lotte intensiviert, desto öfter referiert Werther mittels Gattungsnamen auf sie und nennt sie einen „Engel des Himmels“ und sieht in ihr den Lebenssinn „O der Engel! Um deinetwillen muss ich leben!“ usf. Diese spezifischen Referenzausdrücke auf einzelne Figuren können ausschließlich aus dem Kontext erschlossen werden.

Im Hinblick auf viele Schauplätze und Figuren finden sich teilweise nur vage oder zensierte Angaben (,mit dem Gesandten nach ${ }^{\star \star \star}$ gehen soll“) im Roman, was unter anderem dazu beitrug, dass er in der zeitgenössischen Rezeption als Bezugnahme auf persönliche Erlebnisse des Autors (den Selbstmord Karl Wilhelm Jerusalems und seine Beziehung zur bereits verlobten Charlotte Buff) (Vorderstemann 2007, S. 53, 71) hin gelesen wurde. Für die Annotation hat dieser Kunstgriff Goethes zur Folge, dass sich solche Namensnennungen auf zwei unterschiedliche Entitäten beziehen können: „Graf von M.“, „die übergnädige Dame von S.“. Entsprechend erfolgt hier eine Doppelannotation, um der Verschachtelung von PER und LOC gerecht zu werden. Bei der Annotation der Orte (LOC) kamen ähnliche Fragen wie im mediävistischen Arbeitsbereich auf. Ausgehend von der Intention, durch die Annotation der Orte eine Abfolge dieser sowie eine Gegenüberstellung von Natur- und Stadtraum sichtbar machen zu können, war ebenfalls eine korpusspezifische Eingrenzung notwendig. Sie sollte zeigen, ob ausschließlich Handlungsräume („Lieblingsplätzgen“, „fruchtbare Tal“, „Wirtshaus“) und Städtenennungen („Walheim“) erfasst werden oder ob es notwendig ist, feingranularer zu annotieren, um damit auch Räume und Objekte wie den Tisch oder das Bett Werthers zu erfassen. Als sinnvoll hat sich erwiesen, Objekte nur dann zu annotieren, wenn sich Werther an genau diesem einem ,Brunnen` wiederholt aufhält und er und Lotte in dieser ,Kutsche` zum Ball fahren. Weitere, unbestimmte Nennungen solcher Objekte blieben bei der Annotation unberücksichtigt.

In der Annotationspraxis hat sich darüber hinaus gezeigt, dass die Erfassung von maximalen Nominalphrasen beim Werther eine deutliche Herausforderung darstellt. Der Briefroman ist durch einen expressiven Schreibstil gekennzeichnet, der sich sowohl in einem emotionalen Vokabular manifestiert als auch in der gewollt expressiven Art zu schreiben, die durch unvollständige und verschachtelte Sätze, Parenthesen, Ellipsen und Ausrufe die Emotionalität und Unmittelbarkeit des Geschriebenen unterstreicht (Martens 1985, S. 96). Aus diesem Grund sind 
auch Relativsätze wie „Sie ist schon vergeben, antwortete jene, an [einen sehr braven Mann, der weggereist ist, seine Sachen in Ordnung zu bringen nach seines Vaters Tod, und sich um eine ansehnliche Versorgung zu bewerben] “ keine Besonderheit, bergen jedoch ein hohes Fehlerpotenzial, wenn es darum geht, die exakten Grenzen der maximalen Nominalphrase zu bestimmen (vgl. Abschnitt 2.3).

\subsection{Philosophischer Zugang}

Die Auseinandersetzung mit dem philosophischen Textkorpus im Rahmen von CRETA verfolgte zwei Ziele:

1. die textkritische Edition ausgewählter Überlieferungsträger von Adornos $\ddot{A}$ thetischer Theorie (vgl. Endres et al. 2013 und Adorno o.D.);

2. die computergestützte Textanalyse ausgewählter philosophischer Texte von Adorno.

Zu Förderbeginn von CRETA stand noch nicht fest, welche Fragen an das philosophische Textkorpus gestellt werden sollten. Im Zuge der interdisziplinären Arbeit an einem gemeinsamen Annotationsworkflow bestätigte sich jedoch sehr schnell die Vermutung, dass sich die Philosophie von den anderen beteiligten Disziplinen sowohl in ihrem Umgang mit Entitäten als auch in ihren Textumgangsformen, d.h. den disziplimmanent üblichen Formen der Lektüre und Interpretation von philosophischen Texten, unterscheidet. Der philosophische Umgang mit Entitäten ist am Anfang dieses Aufsatzes bereits kurz skizziert worden. Die Textumgangsformen in der Philosophie unterscheiden sich je nach philosophischem Ansatz. Fast alle dieser Ansätze kennzeichnen sich jedoch gegenwärtig dadurch, dass der Text als für sich stehende und inhaltlich zusammenhängende Folge von Aussagen kaum eine Rolle spielt. ${ }^{22}$ Paradigmatisch zeigt sich dies an demjenigen philosophischen Ansatz, der die Philosophie die letzten Jahrzehnte dominierte: der analytischen Philosophie. Deren Grundannhamen werden exemplarisch in Jay F. Rosenbergs Einführung in die Philosophie artikuliert. ${ }^{23}$ Rosenberg kennzeichnet die Philosophie als eine Disziplin „zweiter Ordnung“, welche Tätigkei-

22 Eine kritische Auseinandersetzung mit derartigen Zugängen sowie etwaige Alternativen bieten Endres et al. 2017.

23 Die deutsche Übersetzung von Rosenbergs 1984 erstmals auf Englisch publizierter Einleitung liegt mittlerweile in der sechsten Auflage vor (Rosenberg 2009). Die Verifikation ihres mutmaßlich paradigmatischen Charakters stellt selbst ein potenzielles DH-Forschungsprojekt dar: Eine Möglichkeit, zu bestimmen, welches Verständnis von Philosophie gegenwärtig dominiert, be- 
ten „erster Ordnung“, wie zum Beispiel Kunst, Literatur, Physik oder Psychologie untersucht. Dabei orientiere sie sich an zwei Gruppen von Fragen: Bedeutungsfragen und Rechtfertigungsfragen. Erstere widmen sich insbesondere der Klärung von Verständnisschwierigkeiten, die sich in Hinblick auf die Behauptungen von Disziplinen ,erster Ordnung ' ergeben können. Letztere untersuchen deren Begründungsmodi. Aus den beiden Fragen und ihren Gegenstandsbereichen lassen sich in Anknüpfung an Rosenberg zwei Praktiken ableiten, welche die Philosophie dominieren: Argumentations- und (Begriffs-)Analyse .

Ein derartiges Philosophieverständnis zeitigt weitreichende Konsequenzen für den Umgang mit philosophischen Texten. Dieser wird sich auf jene Textmerkmale konzentrieren, in welchen sich die argumentative Struktur und der terminologische Gehalt dieser Texte manifestieren. Für den Umgang mit Entitätenreferenzen in philosophischen Texten folgt daraus, dass eine Textanalyse im Sinne von Rosenbergs Philosophieverständnis primär nur an einer der fünf im Rahmen von CRETA entwickelten Entitätenkategorien interessiert sein wird: den abstrakten Konzepten (CNCs). Was ein abstraktes Konzept bzw. ein Begriff ist, ist in der Philosophie jedoch genauso schulabhängig wie das Philosophieverständnis selbst. Daraus folgt, dass, bevor eine textanalytische Fragestellung in Hinblick auf das philosophische Textkorpus entwickelt bzw. präzisiert werden kann, zu klären ist, welcher Begriff des Begriffes dieser zugrundegelegt wird.

Was bedeutete dieser Sachverhalt für den Umgang mit Entitäten und deren Referenzen im Zuge der Auseinandersetzung mit dem philosophischen Textkorpus im Rahmen von CRETA? Obwohl für diese primär die abstrakten Konzepte von Interesse waren, wurde aus den in diesem Aufsatz genannten arbeitsdynamischen und explorativen Gründen der Arbeitsablauf im Umgang mit Entitäten und deren Referenzen aufrechterhalten, d. h., es wurden die Referenzen auf sämtliche der im Zuge von CRETA entwickelten Entitätenkategorien annotiert. ${ }^{24}$ Da jedoch in Adornos Werk die Frage nach dem philosophischen Umgang mit Begriffen selbst eine zentrale Rolle spielt, wurden letztendlich in Anknüpfung an die aktuelle Adornoforschung (vgl. zum Beispiel Hogh 2015) - Adornos Selbstverständnis und dem Fokus der Hauptströmungen der Gegenwartsphilosophie entsprechend - die Be-

steht darin, diejenigen (Lehr-)Bücher zu sammeln, die gegenwärtig in Einführungsveranstaltungen der Philosophie verwendet werden, um zu untersuchen, welches Verständnis von Philosophie in diesen propagiert wird.

24 Dabei bestätigte sich die naheliegende Vermutung, dass auch zwei weitere Entitätenkategorien - Werke und Eigennamen - für historisch-hermeneutische Fragestellungen von Bedeutung sind: So ließe sich über deren Verhältnis zu den abstrakten Konzepten zum Beispiel eruieren, welche Werke welcher Autoren welche Relevanz in den ästhetischen Theorien Adornos und anderer Philosophen besitzen. 
griffe ins Zentrum der konkreten Textanalyse gestellt (vgl. hierzu den Beitrag von Pichler, Blessing et al. 2020 ab Seite 328 in diesem Band).

Mit diesem Fokus wurde jene Frage akut, die bereits am Anfang der manuellen Annotation aufgekommen war: Wie verhält sich die in CRETA etablierte Entitätenkategorie ,abstrakte Konzepte‘ (CNC) zu den Begriffskonzeptionen der Philosophie? Die Beantwortung dieser Frage setzte definitorische Festlegungen voraus, da einerseits in CRETA die Kategorie ,abstrakte Konzepte“ bewusst vage bestimmt wurde - in den Richtlinien ist von „Referenzen auf Konzepte, die für die Analyse wichtig sind“, die Rede - andererseits in der Philosophie selbst - wie bereits angemerkt - keine Einigkeit darüber herrscht, was einen Begriff kennzeichnet. Festzulegen war also erstens, welche Begriffe als für die Analyse relevant erachtet werden, was wiederum, zweitens, eine Klärung dessen voraussetzte, was im Folgenden als Begriff erachtet werden sollte, und wie - drittens - auf diese in den Texten referiert wird.

Die Bestimmung dessen, was bei der Analyse des philosophischen Textkorpus als Begriff verstanden werden sollte, orientierte sich an der im Zuge des Projektes präzisierten disziplinspezifischen Fragestellung. Forschungsziel war es, zu überprüfen, ob Adorno Begriffe seinen Selbstbeschreibungen entsprechend verknüpft und ob sich diese Verknüpfungsform von derjenigen anderer Philosophieschulen unterscheidet. Aus dieser Fragestellung resultierten zwei Anforderung an das zu etablierende Begriffsverständnis: Erstens sollten die Begriffe eine Entitätenkategorie bilden, deren Referenzen sich ohne eine allzu große Anzahl an $\mathrm{Zu}$ satzbestimmungen relativ einfach, wenn möglich sogar automatisch, an der Textoberfläche festmachen und annotieren ließen. Zweitens galt es, eine Konzeptualisierung von ,Begriff‘ zu entwickeln, die keine der Begriffskonzeptionen, welche die in das Textkorpus aufgenommenen Philosophien vertraten, bevorzugte. Nur so konnte vermieden werden, dass die Beantwortung der leitenden Fragestellung in eine zirkuläre Argumentation mündete und damit als typisches Resultat eines confirmation bias erachtet werden würde.

Bei der Suche nach einer diesen Voraussetzungen entsprechenden Begriffskonzeption zeigte sich, dass eine Übernahme des in der sprachanalytisch orientierten Gegenwartsphilosophie dominierenden Verständnisses von Begriffen nicht nur eine umfangreiche Ergänzung der in CRETA entwickelten Annotationsrichtlinien von Nöten gemacht hätte, sondern auch von Vorannahmen geprägt ist, gegen die sich Adorno mit seinem Denken wandte. ${ }^{25}$ Die sprachanalytisch orientierte Philosophie unterscheidet - grob gesagt - zwischen singulären Begrif-

25 Adorno wandte sich insbesondere gegen den vermeintlichen ,Zwangscharakter‘ eines an der formalen Logik orientierten Denkens, vgl. zum Beispiel Adorno 2003. 
fen, die ein konkretes Einzelding bezeichnen (z. B. Eigennamen), und generellen Begriffen bzw. Termini, die durch ihre Anwendung auf singuläre Begriffe letztere bestimmen, indem sie atomare Sätze bilden (z. B.: „Sokrates ist ein Philosoph“). Generelle Termini werden daher auch als Prädikate bezeichnet. Bezüglich derselben können Eigenschaftsbegriffe (z. B.: „X ist grün“) von Artbegriffen (z. B.: „X ist ein Philosoph“) unterschieden werden. Generelle Termini können sowohl durch Nomen und Adjektive als auch durch Verben sprachlich realisiert werden (z. B.: ,x liebt y“). Dieser Sachverhalt sowie die Tatsache, dass es sich bei generellen Termini um Funktionsausdrücke handelt, für die das Relationsverhältnis von zentraler Bedeutung ist, erlauben zwar deren formale Transkription, erschweren jedoch zugleich ihre Auszeichnung auf der Textoberfläche. Letzteres hätte, wie bereits angemerkt, eine Erweiterung der CRETA-Annotationsrichtlinen notwendig gemacht. Abgesehen von diesen pragmatischen Gründen erschien es nicht sinnvoll, für ein Begriffsverständnis und die damit einhergehende Form der Begriffsverknüpfung, für die bereits eine etablierte Formalisierungspraxis existiert - die Prädikatenlogik -, eine alternative Form zu entwickeln.

In Anbetracht dieser Gründe wurde letztendlich auf ein Begriffsverständnis zurückgegriffen, das sich nahe am alltäglichen Verständnis von ,Begriff‘ bewegt und das zudem problemlos in die CRETA-Entitätenkonzeption integriert werden konnte: Als Referenzen auf Begriffe wurden sämtliche Nomina erachtet, bei denen es sich nicht um eine der vier anderen in CRETA entwickelten Entitätenkategorien handelte.

Abschließend ist festzuhalten, dass sich im philosophischen Arbeitsbereich disziplinspezifische Vorannahmen und damit einhergehende Textumgangsformen weitaus stärker auf die Arbeit mit den in CRETA entwickelten Entitätenklassen auswirkten als bei den anderen hier vorgestellten Arbeiten. Diese Vorannahmen sowie die im Zuge des Projektes entwickelte Fragestellung führten letztendlich dazu, dass nur eine der fünf CRETA-Entitätenklassen als heuristisch relevant erschien - und auch das erst, nachdem diese Klasse, die abstrakten Konzepte (CNC), der Disziplin entsprechend präzisiert wurde. Diese Präzisierung und die mit ihr einhergehende Begriffsarbeit führte zu einer Selbstreflexion zentraler Vorannahmen der Auseinandersetzung mit philosophischen Texten, wie sie bei der Interpretation philosophischer Texte nicht immer üblich ist, und generierte so einen zusätzlichen Erkenntnisgewinn für das Projekt. Dieser Mehrwert ist eine Folge des interdisziplinären Dialogs sowie der interdisziplinären Arbeit an einem gemeinsamen Workflow, ohne die die zu ihm führenden Reflexionen wahrscheinlich gar nicht erst angestoßen worden wären. 


\section{Schlussbetrachtung}

Der in CRETA verfolgte und in diesem Artikel beschriebene Ansatz ging von der Annahme aus, dass mit der Annotation von Entitäten ein Konzept in den Blick genommen wird, das für alle beteiligten Disziplinen von Relevanz und für disziplinspezifische Fragestellungen anschlussfähig ist. Dabei standen zwei Ziele im Fokus: Erstens sollte durch den interdisziplinären Austausch eine methodische Umsetzung entwickelt werden, die im Gesamten oder zumindest in Teilen disziplinenunabhängig einsetzbar ist und so dazu beiträgt, eine disziplinenunabhängige Begrifflichkeit für CRETA zu entwickeln. Zweitens sollte die Auseinandersetzung mit Entitäten und ihren Referenzen zur Entwicklung und Schärfung der disziplinären Fragestellungen beitragen. Beide Zielsetzungen werden hier abschließend evaluiert und reflektiert.

In Hinblick auf die einheitliche methodische Umsetzung lässt sich zunächst festhalten, dass die enge Zusammenarbeit es ermöglicht hat, sowohl unterschiedliche Entitätenklassen disziplinübergreifend in Annotationsrichtlinien zu definieren als auch einen gemeinsamen Arbeitsablauf für die manuelle und semiautomatische Annotation zu entwickeln. Dabei ist allerdings anzumerken, dass die Annotationsrichtlinien zunächst wiederholt auf die verschiedenen Textkorpora angewendet werden mussten, um text- bzw. disziplinspezifische Konkretisierungen oder Anpassungen vorzunehmen. Die notwendigen Ergänzungen reichten von der Hinzunahme weiterer Referenzausdrücke (etwa Pronomina im Werther-Korpus) über die Berücksichtigung zusätzlicher Entscheidungskriterien (z. B. die Unterscheidung von fiktiver Figur und historischer Person) bis hin zur Implementierung weiterer umfassender Arbeitsschritte (entity grounding). Die zunächst tendenziell generischen Annotationsrichtlinien waren jedoch grundlegend für die disziplinären Spezifikationen und erleichterten diese erheblich. Die bei der Entitätenannotation entwickelten Arbeitsabläufe erwiesen sich somit für alle Disziplinen als effektiv und bestätigten somit zentrale methodische Grundannahmen von CRETA (vgl. auch den Beitrag von Pichler und Reiter 2020 ab Seite 43 in diesem Band).

Die wiederholte Auseinandersetzung mit dem Konzept der Entität und ihrer Referenz trug darüber hinaus zu einem tieferen Verständnis innerhalb der einzelnen Disziplinen bei. Dabei erwies sich insbesondere der Prozess der Parallelannotation als fruchtbar, den disziplininternen Dialog zu fördern, Vorannahmen offenzulegen und sie dadurch zur Diskussion zur stellen. Zudem ermöglichte die unmittelbare Anwendung der Annotationsrichtlinien auf die jeweiligen Textkorpora, die fachspezifischen Fragestellungen so zu präzisieren, dass eine Operationalisierung und Beantwortung dieser im Zuge einer reflektierten algorithmischen 
Textanalyse möglich war. Der semi-automatische Annotationsprozess (vgl. Abbildung 4) ist zwar (bisher) nur für das mediävistische Projekt zum Einsatz gekommen, er wurde in diesem Kontext jedoch bereits auf verschiedene Texte und Textgattungen angewendet, was nahelegt, dass er auch ohne Weiteres auf Texte anderer Disziplinen übertragen werden kann.

In summa profitierten sämtliche Teilnehmenden von den Synergieeffekten des interdisziplinären Dialogs (vgl. hierzu auch den Beitrag von Reiter, Kremer et al. (2020), ab Seite 467 in diesem Band). Zwar wurde im Laufe des Projektes deutlich, dass alle Fachbereiche von disziplinspezifischen Vorannahmen geprägt waren, die einen bestimmten Umgang mit den Untersuchungsgegenständen nahelegen und zum Verständnis aller wiederholt diskutiert wurden. Dieser vermeintliche Nachteil schlug jedoch in einen Vorteil um, da die interdisziplinäre Kooperation die Teilnehmenden von Anfang an dazu verpflichtete, besagte Vorannahmen offenzulegen, wodurch sich ein gegenseitiges Verständnis bilden konnte. Denn nur unter Berücksichtigung derselben konnte die Entwicklung eines interdisziplinären Entitätenkonzeptes und diesem entsprechender Entitätenklassen sowie eines damit einhergehenden Annotationsworkflows realisiert werden. Diese Form des Austauschs trug letztendlich sowohl zur Etablierung neuer Arbeitspraktiken und Methoden als auch zu einem vertieften Selbstverständnis der teilnehmenden Disziplinen bei. Insofern hat sich das Vorgehen für alle Disziplinen als fruchtbar erwiesen und eignet sich darüber hinaus als methodische Grundlage für zukünftige Kooperationen an der Schnittstelle von Sozial- und Geisteswissenschaften einerseits und Computerlinguistik andererseits.

Danksagung: Wir bedanken uns bei Florian Barth, Dilan Cakir, Anaïck Geissel, Annika Holzer, Alina Palesch, Fabian Schan, Thomas Frank und Jan Velimsky für ihre wertvolle Hilfe bei der manuellen Annotation.

\section{Primärliteratur}

Goethe, Johann Wolfgang (1899). Die Leiden des jungen Werther. I. A. d. Großherzogin Sophie von Sachsen. Weimar: Hermann Böhlaus Nachfolger.

Hartmann von Aue (1963). Erec. Hrsg. von Albert Leitzmann. 3. Aufl. Tübingen: Niemeyer. Hartmann von Aue (1968). Iwein. Hrsg. von Georg F. Benecke, Karl Lachmann und Ludwig Wolff. 7. Aufl. Berlin: de Gruyter.

Wolfram von Eschenbach (1891). Parzival. Hrsg. von Karl Lachmann. 5. Aufl. Berlin. 


\section{Sekundärliteratur}

Adorno, Theodor W. (2003). „Einleitung zum Positivismusstreit in der deutschen Soziologie“. In: Hrsg. von Rolf Tiedemann, Gretel Adorno, Susan Buck-Morss und Klaus Schultz. Bd. 8. Gesammelte Schriften. Frankfurt am Main: Suhrkamp, S. 280-353.

Adorno, Theodor W. (o.D.). Schein - Form - Subjekt - Prozeßcharakter - Kunstwerk. Textkritische Edition der letzten bekannten Überabreitung des III. Kapitels der 'Kapitel-Ästhetik'. Hrsg. von Martin Endres, Axel Pichler und Claus Zittel.

Appell, Johann Wilhelm (1882). Werther und seine Zeit. Oldenburg: Schulzesche Hofbuchhandlung.

Atkins, Stuart P. (1949). The testament of Werther in poetry and drama. Cambridge, Massachusetts: Harvard University Press.

Barth, Florian und Sandra Murr (2017). „Digital Analysis of the Literary Reception of J.W. von Goethe’s Die Leiden des jungen Werthers“. In: Digital Humanities 2017: Conference Abstracts. Montreal, S. 540-542.

Barth, Florian und Gabriel Viehhauser (2017). „Digitale Modellierung literarischen Raum“. In: Abstracts der DHd: Digitale Nachhaltigkeit. Bern: Digital Humanities im deutschsprachigen Raum e.V., S. 128-132.

Baumann, Martin, Steffen Koch, Markus John und Thomas Ertl (2020). „Interactive Visualization for Reflected Text Analytics“. In: Reflektierte Algorithmische Textanalyse. Hrsg. von Nils Reiter, Axel Pichler und Jonas Kuhn. Berlin: De Gruyter, S. 270-296.

Bethard, Steven, Philip Ogren und Lee Becker (2014). „ClearTK 2.0: Design Patterns for Machine Learning in UIMA“. In: Proceedings of the Ninth International Conference on Language Resources and Evaluation (LREC'14). Reykjavik, Iceland: European Language Resources Association (ELRA), S. 3289-3293.

Blätte, Andreas und André Blessing (2018). „The GermaParl Corpus of Parliamentary Protocols“. In: Proceedings of the Eleventh International Conference on Language Resources and Evaluation (LREC 2018), S. 810-816.

Blessing, André, Nora Echelmeyer, Markus John und Nils Reiter (2017). „An End-to-end Environment for Research Question-Driven Entity Extraction and Network Analysis“. In: Proceedings of the Joint SIGHUM Workshop on Computational Linguistics for Cultural Heritage, Social Sciences, Humanities and Literature. Vancouver, Canada: Association for Computational Linguistics, S. 57-67. DoI: 10.18653/v1/W17-2208.

Braun, Manuel und Nora Ketschik (2019). „Soziale Netzwerkanalysen zum mittelhochdeutschen Artusroman oder: Vorgreiflicher Versuch, Märchenhaftigkeit des Erzählens zu messen“. In: Das Mittelalter 24, S. 54-70. Dol: 10.1515/mial-2019-0005.

Carstensen, Kai-Uwe, Christian Ebert, Cornelia Ebert, Susanne Jekat, Ralf Klabunde und Hagen Langer, Hrsg. (2010). Computerlinguistik und Sprachtechnologie. Eine Einführung. Heidelberg: Spektrum. DoI: 10.1007/978-3-8274-2224-8.

Clark, Alexander, Chris Fox und Shalom Lappin, Hrsg. (2013). The handbook of computational linguistics and natural language processing. Chichester: Wiley-Blackwell.

Cohen, Jacob (1960). „A Coefficient of Agreement for Nominal Scales“. In: Educational and Psychological Measurement 20.1, S. 37-46.

Echelmeyer, Nora, Nils Reiter und Sarah Schulz (2017). „Ein PoS-Tagger für ,das‘ Mittelhochdeutsche“. In: Abstracts der DHd: Digitale Nachhaltigkeit. Bern: Digital Humanities im deutschsprachigen Raum e.V., S. 141-147. 
Endres, Martin, Axel Pichler und Claus Zittel (2013). „,Noch offen‘. Prolegomena zu einer Textkritischen Edition der Ästhetischen Theorie Adornos“. In: editio 27, S. 173-204.

Endres, Martin, Axel Pichler und Claus Zittel, Hrsg. (2017). Textologie. Theorie und Praxis interdisziplinärer Textforschung. Berlin: De Guyter.

Faruqui, Manaal und Sebastian Padó (2010). „Training and Evaluating a German Named Entity Recognizer with Semantic Generalization“. In: Proceedings of KONVENS, S. 129-133.

Finkel, Jenny Rose, Trond Grenager und Christopher Manning (2005). „Incorporating Non-local Information into Information Extraction Systems by Gibbs Sampling“. In: Proceedings of the 43nd Annual Meeting of the Association for Computational Linguistics. Ann Arbor, Michigan, USA: Association for Computational Linguistics, S. 363-370. Dol: 10.3115 / 1219840.1219885.

Forster, Edward Morgan (1949). Ansichten des Romans. Übers. von Walter Schürenberg. Frankfurt am Main: Suhrkamp.

Gius, Evelyn und Janina Jacke (2017). „The Hermeneutic Profit of Annotation. On preventing and fostering disagreement in literary text analysis“. In: International Journal of Humanities and Arts Computing 11.2, S. 233-254.

Goedeke, Karl und Edmund Goetze (1979). Grundriss zur Geschichte der deutschen Dichtung aus den Quellen. Nendeln, Lichtenstein: Kraus Reprint.

Haferland, Harald (2013). „Psychologie und Psychologisierung: Thesen zur Konstitution und Rezeption von Figuren. Mit einem Blick auf ihre historische Differenz“. In: Erzähllogiken in der Literatur des Mittelalters und der Frühen Neuzeit. Akten der Heidelberger Tagung vom 17. bis 19. Februar 2011. Hrsg. von Florian Kragl und Christian Schneider. Heidelberg: Winter, S. 91-117.

Hogh, Philip (2015). Kommunikation und Ausdruck: Sprachphilosophie nach Adorno. Weilerswist: Velbrück.

Horré, Thomas (1997). Werther-Roman und Werther-Figur in der deutschen Prosa des Wilhelminischen Zeitalters. St. Ingbert: Röhrig.

Jansen, Dorothea (2003). Einführung in die Netzwerkanalyse. Grundlagen, Methoden, Forschungsbeispiele. Opladen: Leske \& Budrich. Dol: 10.1007/978-3-663-09875-1.

Jurafsky, Daniel und James H. Martin (2008). Speech and language processing. An introduction to natural language processing. 2. Aufl. Upper Saddle River, NJ: Prentice Hall.

Kantner, Cathleen (2004). Kein modernes Babel: Kommunikative Voraussetzungen europäischer Öffentlichkeit. Wiesbaden: Springer VS.

Kantner, Cathleen (2015). War and Intervention in the Transnational Public Sphere: Problemsolving and European identity-formation. London/New York: Routledge.

Kantner, Cathleen und Udo Tietz (2013). „Identitäten und multiple Identitäten. Über die wertrationale Integration der Gemeinschaften unter den Bedingungen der Moderne“. In: Dialektik-Arbeit-Gesellschaft. Festschrift für Peter Ruben. Hrsg. von Erhard Crome und Udo Tietz. Potsdam: Welt-Trends, S. 47-63.

Ketschik, Nora (in Vorbereitung). „Mittelhochdeutsche Großepik im Lichte computergestützter Methoden (Arbeitstitel)“. Diss. Universität Stuttgart.

Kuhn, Jonas (2020). „Einleitung“. In: Reflektierte Algorithmische Textanalyse. Hrsg. von Nils Reiter, Axel Pichler und Jonas Kuhn. Berlin: De Gruyter, S. 9-40.

Lafferty, John, Andrew McCallum und Fernando C. N. Pereira (2001). „Conditional Random Fields: Probabilistic Models for Segmenting and Labeling Sequence Data“. In: Proceedings of the Eighteenth International Conference on Machine Learning. Burlington/San Francisco: Morgan Kaufmann Publishers, S. 282-289. 
Lahn, Silke und Jan Christoph Meister (2016). Einführung in die Erzähltextanalyse. 3. Aufl. Stuttgart: Metzler.

Lotman, Juri M. (1972). Die Struktur literarischer Texte. Übers. von Rolf-Dietrich Keil. München/Stuttgart: UTB.

Manning, Christopher und Hinrich Schütze (1999). Foundations of Statistical Natural Language Processing. 2. Aufl. Cambridge, Massachusetts: MIT Press.

Martens, Lorna (1985). The Diary Novel. Cambridge: Cambridge University Press.

McCallum, Andrew Kachites (2002). „MALLET: A Machine Learning for Language Toolkit“. http://mallet.cs.umass.edu. (Besucht am 1. Juni 2020).

Pfister, Manfred (2001). Das Drama: Theorie und Analyse. 11. Aufl. Bd. 580. Uni-Taschenbücher Literaturwissenschaft. München: Fink.

Pichler, Axel, André Blessing, Nils Reiter und Mirco Schönfeld (2020). „Algorithmische Mikrolektüren philosophischer Texte". In: Reflektierte Algorithmische Textanalyse. Hrsg. von Nils Reiter, Axel Pichler und Jonas Kuhn. Berlin: De Gruyter, S. 328-372.

Pichler, Axel und Nils Reiter (2020). „Reflektierte Textanalyse“. In: Reflektierte Algorithmische Textanalyse. Hrsg. von Nils Reiter, Axel Pichler und Jonas Kuhn. Berlin: De Gruyter, S. 4359.

Platz-Waury, Elke (1997a). „Figur“. In: Reallexikon der deutschen Literaturwissenschaft. Neubearbeitung des Reallexikons der deutschen Literaturgeschichte. Hrsg. von Klaus Weimar. Berlin: De Gruyter, S. 587-589.

Platz-Waury, Elke (1997b). „Figurenkonstellation“. In: Reallexikon der deutschen Literaturwissenschaft. Neubearbeitung des Reallexikons der deutschen Literaturgeschichte. Hrsg. von Klaus Weimar. Berlin: De Gruyter, S. 591-593.

Propp, Vladimir Jakovlevič (1972). Morphologie des Märchens. Russisch übers. von Karl Eimermacher. München: Hanser.

Reiter, Nils (2020). „Anleitung zur Erstellung von Annotationsrichtlinien“. In: Reflektierte Algorithmische Textanalyse. Hrsg. von Nils Reiter, Axel Pichler und Jonas Kuhn. Berlin: De Gruyter, S. 193-201.

Reiter, Nils, André Blessing, Nora Echelmeyer, Steffen Koch, Gerhard Kremer, Sandra Murr und Max Overbeck (2017). „CRETA Unshared Task zu Entitätenreferenzen (CUTE)“. In: Abstracts der DHd: Digitale Nachhaltigkeit. Bern: Digital Humanities im deutschsprachigen Raum e.V., S. 19-22.

Reiter, Nils, Gerhard Kremer, Kerstin Jung, Benjamin Krautter, Janis Pagel und Axel Pichler (2020). „Reaching out: Interdisziplinäre Kommunikation und Dissemination“. In: Reflektierte Algorithmische Textanalyse. Hrsg. von Nils Reiter, Axel Pichler und Jonas Kuhn. Berlin: De Gruyter, S. 467-484.

Reznicek, Marc (2013a). „Linguistische Annotation von Nichtstandardvarietäten - Guidelines und 'Best Practices'. Guidelines Koreferenz. Version 1.1'. Annotationsrichtlinien. Humboldt-Universität zu Berlin. URL: https: / / www.linguistik. hu-berlin.de/de/institut/ professuren/korpuslinguistik / forschung/nosta- $d$ /nosta- $d$ - cor-1.1 (besucht am 1. Juni 2020).

Reznicek, Marc (2013b). „Linguistische Annotation von Nichtstandardvarietäten - Guidelines und 'Best Practices'. Guidelines NER. Version 1.5“. Annotationsrichtlinien. HumboldtUniversität zu Berlin. URL: https://www.linguistik.hu-berlin.de/de/institut/professuren/ korpuslinguistik/forschung/nosta-d/nosta-d-ner-1.5 (besucht am 1. Juni 2020).

Richter, Sandra (2017). Eine Weltgeschichte der deutschsprachigen Literatur. München: C. Bertelsmann Verlag. 
Rorty, Richard M. (1992). The Linguistic Turn. Essays in Philosophical Method. University of Chicago Press: Chicago.

Rosenberg, Jay (2009). Philosophieren. Ein Handbuch für Anfänger. Übers. von Brigitte Flickinger. 6. Aufl. Frankfurt am Main: Vittorio Klostermann.

Scherpe, Klaus R. (1970). Werther und Wertherwirkung. Zum Syndrom bürgerlicher Gesellschaftsordnung im 18. Jahrhundert. Bad Homburg: Athenaion.

Schulz, Armin (2012). Erzähltheorie in mediävistischer Perspektive. Berlin: De Gruyter.

Schulz, Sarah (2018). „The Taming of the Shrew. Non-standard text processing in the Digital Humanities “. Diss. Universität Stuttgart. DoI: 10.18419/opus-9685.

Tietz, Udo (2002). Die Grenzen des "Wir". Eine Theorie der Gemeinschaft. Frankfurt am Main: Suhrkamp.

Trilcke, Peer (2013). „Social Network Analysis (SNA) als Methode einer textempirischen Literaturwissenschaft“. In: Empirie in der Literaturwissenschaft. Hrsg. von Philip Ajouri, Christoph Rauen und Katja Mellmann. Bd. 8. Poetogenesis - Studien zur empirischen Anthropologie der Literatur. Münster: Mentis, S. 201-247. DoI: 10.30965/9783957439710_012.

Viehhauser, Gabriel (2020). „Zur Erkennung von Raum in narrativen Texten“. In: Reflektierte Algorithmische Textanalyse. Hrsg. von Nils Reiter, Axel Pichler und Jonas Kuhn. Berlin: De Gruyter, S. 373-388.

Vorderstemann, Karin (2007). Ausgelitten hast du - ausgerungen ...": Lyrische Wertheriaden im 18. und 19. Jahrhundert. Heidelberg: Winter. 